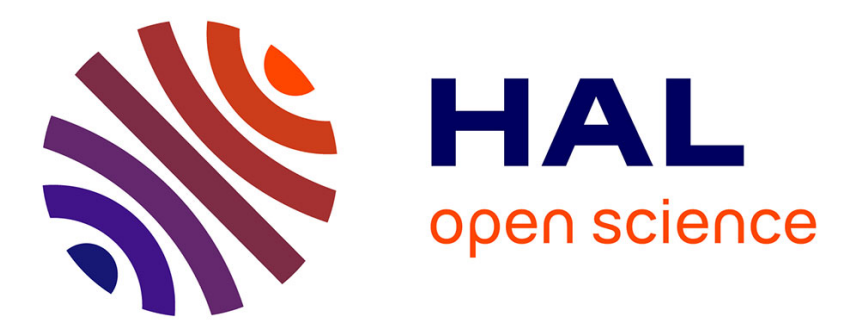

\title{
Birth and life of auroral arcs embedded in the evening auroral oval convection: A critical comparison of observations with theory
}

Gerhard Haerendel, Harald U. Frey, Christopher C. Chaston, Olaf Amm, Liisa Juusola, R. Nakamura, Elena Seran, J. M. Weygand

\section{To cite this version:}

Gerhard Haerendel, Harald U. Frey, Christopher C. Chaston, Olaf Amm, Liisa Juusola, et al.. Birth and life of auroral arcs embedded in the evening auroral oval convection: A critical comparison of observations with theory. Journal of Geophysical Research Space Physics, 2012, 117 (A12), pp.A12220. 10.1029/2012JA018128. hal-00776086

\section{HAL Id: hal-00776086 https://hal.science/hal-00776086}

Submitted on 22 Jan 2017

HAL is a multi-disciplinary open access archive for the deposit and dissemination of scientific research documents, whether they are published or not. The documents may come from teaching and research institutions in France or abroad, or from public or private research centers.
L'archive ouverte pluridisciplinaire HAL, est destinée au dépôt et à la diffusion de documents scientifiques de niveau recherche, publiés ou non, émanant des établissements d'enseignement et de recherche français ou étrangers, des laboratoires publics ou privés. 


\title{
Birth and life of auroral arcs embedded in the evening auroral oval convection: A critical comparison of observations with theory
}

\author{
G. Haerendel, ${ }^{1}$ H. U. Frey, ${ }^{2}$ C. C. Chaston, ${ }^{2}$ O. Amm, ${ }^{3}$ L. Juusola, ${ }^{3}$ R. Nakamura, ${ }^{4}$ \\ E. Seran, ${ }^{5}$ and J. M. Weygand ${ }^{6}$
}

Received 13 July 2012; revised 23 October 2012; accepted 23 October 2012; published 14 December 2012.

[1] We present and analyze data on auroral arcs obtained during a pass of the FAST satellite over the field-of-view of the all-sky camera at Ft. Simpson (Canada), supported by ground-based magnetometer and SuperDARN radar data, and plasma data from THEMIS-A near the source region of the auroral currents. The auroral event took place at 19:00 MLT during substorm activity further east. Active auroral arcs were present over six degrees in latitude moving equatorward with significant changes in brightness and structure. New arcs were forming continuously at the polar border of the auroral oval which was marked by an Alfvénic arc. The data analysis revealed that the equatorward drift of the arcs was in part due to convective motion of the plasma frame but was rather dominated by proper motions of the arcs. Interpretation of these findings in the framework of theoretical work by one of the authors reproduces quantitatively the observed proper motion as a consequence of the progressive erosion of magnetic shear stresses. Most important was the possibility to deduce the interaction time scale between arc and source region. On average it corresponded to about six to eight transit times of an Alfvén wave between arc and source plasma or two fundamental eigenperiods of toroidal mode or azimuthally polarized Alfvén waves.

However, large variations of the interaction times and corresponding proper motions were found. They are attributed to temporal and spatial variations of the energy input from the source plasma. The more remarkable is the fact that analysis on the basis of a quasistationary model produces consistent results. The progressive release of shear stresses during the equatorward motion of the arcs leads to the conclusion that they are dying after having reached the maximum of the poleward Pedersen current.

Citation: Haerendel, G., H. U. Frey, C. C. Chaston, O. Amm, L. Juusola, R. Nakamura, E. Seran, and J. M. Weygand (2012), Birth and life of auroral arcs embedded in the evening auroral oval convection: A critical comparison of observations with theory, J. Geophys. Res., 117, A12220, doi:10.1029/2012JA018128.

\section{Introduction}

[2] An enormous wealth of data exists on auroral arcs obtained by various ground-based and space-borne observing techniques. A gross understanding of the plasma origin of the fascinating visual appearances and accompanying geomagnetic and ionospheric effects has been obtained [cf. Paschmann et al., 2002]. All the same few direct investigations have been carried out on the large-scale connections between source plasma, global current system, and the

\footnotetext{
${ }^{1}$ Max Planck Institute for Extraterrestrial Physics, Garching, Germany. ${ }^{2}$ Space Sciences Laboratory, University of California, Berkeley, California, USA.

${ }^{3}$ Arctic Research Unit, Finnish Meteorological Institute, Helsinki, Finland.

${ }^{4}$ Space Research Institute, Austrian Academy of Sciences, Graz, Austria.

${ }^{5}$ LATMOS, IPSL, St.-Maur des Fosses, France.

${ }^{6}$ IGPP, University of California, Los Angeles, California, USA.

Corresponding author: G. Haerendel, Max Planck Institute for Extraterrestrial Physics, D-85748 Garching, Germany. (hae@mpe.mpg.de)

(C2012. American Geophysical Union. All Rights Reserved. 0148-0227/12/2012JA018128
}

energy conversion above and inside the ionosphere [e.g., Marghitu et al., 2006; Hamrin et al., 2006; Frey et al., 2010]. By contrast much theoretical work has been done on the so-called M-I coupling, i.e., the interaction of the hot magnetospheric plasma with the ionosphere. There are for instance ionospheric feedback models [Sato, 1978; Lysak and Song, 2002; Hasegawa et al., 2010], models based on field line resonances [e.g., Rankin et al., 1999; Streltsov and Lotko, 1995; Lotko et al., 1998], treatments of inertial Alfvén waves [Seyler, 1990; Lysak and Lotko, 1996], and Alfvén wave models for the acceleration region [Lysak and Dum, 1983; Haerendel, 1980, 2007; Echim et al., 2009]. Although plenty of in situ data on Alfvén waves exist [e.g., Wygant et al., 2000; Keiling et al., 2003], most of the studies have been carried out within the local environment.

[3] Another less explored aspect is the evolution of auroral arcs. Oguti [1974, 1975] pioneered the classification of structural transformations of arcs like rotations, splitting and unfolding, while Hallinan and Davis [1970] were the first to describe auroral rays as vortex streets. Haerendel et al. [1993] discovered the proper motions of arcs in the plasma frame. De la Beaujardière et al. [1994] observed the 
appearance of a new arc near the poleward auroral boundary and the equatorward motion of an already present arc, probably an expression of such proper motion. Elphinstone et al. [1996] presented a comprehensive overview of the wide variety of large-scale auroral forms during substorms. Karlsson et al. [2004] used the four Cluster satellites near perigee in a pearls-on-a-string fashion to separate temporal and spatial evolutions of auroral current sheets. These are only a few examples from a wide literature on the dynamics of auroral arcs. However, little has been done in relating the observations to the wider plasma and field environment. One of the few examples is the study of small and meso-scale properties of an arc by the Reimei and THEMIS satellites and ground-based data by Frey et al. [2010].

[4] The reason for the lack of coordinated ground-based and satellite investigations of auroral arcs lies in the rarity of having near simultaneous auroral images, clear skies, and the desired satellite passing overhead or being close to the source region of the auroral currents. For this reason, some have even undertaken the effort to fly auroral cameras on airplanes, thereby removing the problem of cloud cover [e.g., Stenbaek-Nielsen et al., 1998]. The alternative of installing imagers on the spacecraft usually does not allow detailed studies of individual arcs because of the limited spatial resolution, the poor cadence of imaging, and the fast motion of the spacecraft. The Japanese satellite, Reimei, overcame much of these drawbacks by its low orbit and active pointing of the camera toward the magnetic foot points [e.g., Sakanoi et al., 2003; Frey et al., 2010]. On the other hand, the low orbit meant to forego the possibility of intersecting the auroral acceleration regions, not to speak of the absence of an onboard magnetometer and electric field instrument.

[5] Since 2006 the situation has greatly changed thanks to the great number and wide spatial distribution of the THEMIS Ground-Based Observatories (GBO) network with panchromatic all-sky cameras and magnetometers and the high cadence of the all-sky imaging [Mende et al., 2008; Russell et al., 2008]. This is especially useful if the observed arc or system of arcs is very active and moving during the short interval of satellite crossing. The extended temporal coverage of the same objects by ground-based imaging adds a dimension that can never be covered by satellite measurements.

[6] The crossing of the field-of-view (FOV) of the all sky camera at Ft. Simpson, Canada, (FSIM) by the FAST spacecraft on 09 March 2008 was one of several events suited for detailed study of dynamical auroral arcs. There were several continually evolving arcs overhead moving generally equatorward and westward. The main focus of this investigation is on the motions and evolution of the arcs including their birth and death. The availability of particle and magnetic field data allows us, in addition, to draw conclusions on the state of the magnetospheric region feeding the energy consumed by the arcs. The fortuitous circumstance that one of the THEMIS spacecraft was in the proximity of the highaltitude plasma connected with the arcs provides a rare opportunity for a cross check.

\section{Ground-Based and Satellite Observations}

[7] The provisional AE index and also the THEMIS pseudo-AE Index shown in Figure 1 (first panel) for 09 March 2008 show the onset of strong substorm activity around 02 UT with intensification around $04 \mathrm{UT}$, the time of the FAST crossing of FSIM. However, at a magnetic local time of about 19:00 (03:58 UT) Ft. Simpson was outside the substorm bulge even though strong westward convection along the oval and auroral activity was present. The westward traveling surge reached the FOV of the all sky camera at the neighboring station of Ft. Smith FSMI at 04:14 UT, i.e., 10 min after the FAST crossing at FSIM.

\subsection{The THEMIS GBO All-Sky Imagers and Ground Magnetometers}

[8] The THEMIS project includes an array of 21 GroundBased Observatories (GBO) with panchromatic all-sky cameras (ASI) and magnetometers [Mende et al., 2008; Russell et al., 2008]. The ASI run at $3 \mathrm{~s}$ cadence with one second integrations and the magnetometers run at $2 \mathrm{~Hz}$. Here we concentrate on the ASI stations Fort Simpson (FSIM, geographic latitude/longitude $61.8 / 238.8^{\circ}$, geomagnetic $67.3 /$ $293.8^{\circ}$ ) and Fort Smith (FSMI, geographic 60.0/248.1 ${ }^{\circ}$, geomagnetic $67.4 / 306.6^{\circ}$ ).

\subsubsection{Magnetometer Observations}

[9] Figure 1 shows the horizontal components of ground magnetometers in central Canada during the first hours of March 9, 2008. The THEMIS pseudo-AE slowly rises after 2:00 UT consistent with enhanced auroral activity in eastern Canada, but in the region of interest there is not much distinct substorm activity until after 04:14 UT when a Westward Traveling Surge (WTS) arrived at the station FSMI. The geomagnetic $\mathrm{H}$-components are distinctively different between the more poleward stations with negative deflections, and the more equatorward stations with positive deflections. For the preceding hours FSIM was recording positive H, i.e., an eastward electrojet. However, at the time of the FAST passage, $\mathrm{H}$ at FSIM was transiting from positive to negative. The bottom plot shows the vertical component of the magnetic disturbance at FSIM with strong negative values during the preceding two hours and beyond. Therefore the center of the ionospheric Hall current was located south of FSIM during the event under investigation.

\subsubsection{All-Sky Camera Observations}

[10] The 9th March 2008 was a moderately disturbed day. At the time of interest around 4:00 UT the Dst reached $-30 \mathrm{nT}$ and the AE index reached $900 \mathrm{nT}$, with extreme values for the day occurring two hours later with -70 and $1300 \mathrm{nT}$, respectively. The local time at the FSIM station was 1900 MLT and an unusually active aurora was observed at that early evening local time. Six images shown in Figure 2 cover essentially the interval of FAST crossing the FOV of the all-sky camera at FSIM. The poleward border of the visible auroral oval was about $200 \mathrm{~km}$ to the north of FSIM but still very well covered by the all-sky imager. Several individual auroral arcs could be identified and according to their relative location they are numbered $1-6$ in Table 1 with arc 1 being the most poleward one.

[11] The keograms in Figure 3 summarize the overall situation and the long-term trend of the auroral evolution. Both FSIM and FSMI cover the poleward edge of the visible auroral oval quite well while it is far too poleward for GILL and equatorward for YKNF. YKNF only covers the later poleward substorm expansion. Up to $4: 10$ UT the most poleward auroral arc, marking the equatorward boundary of 


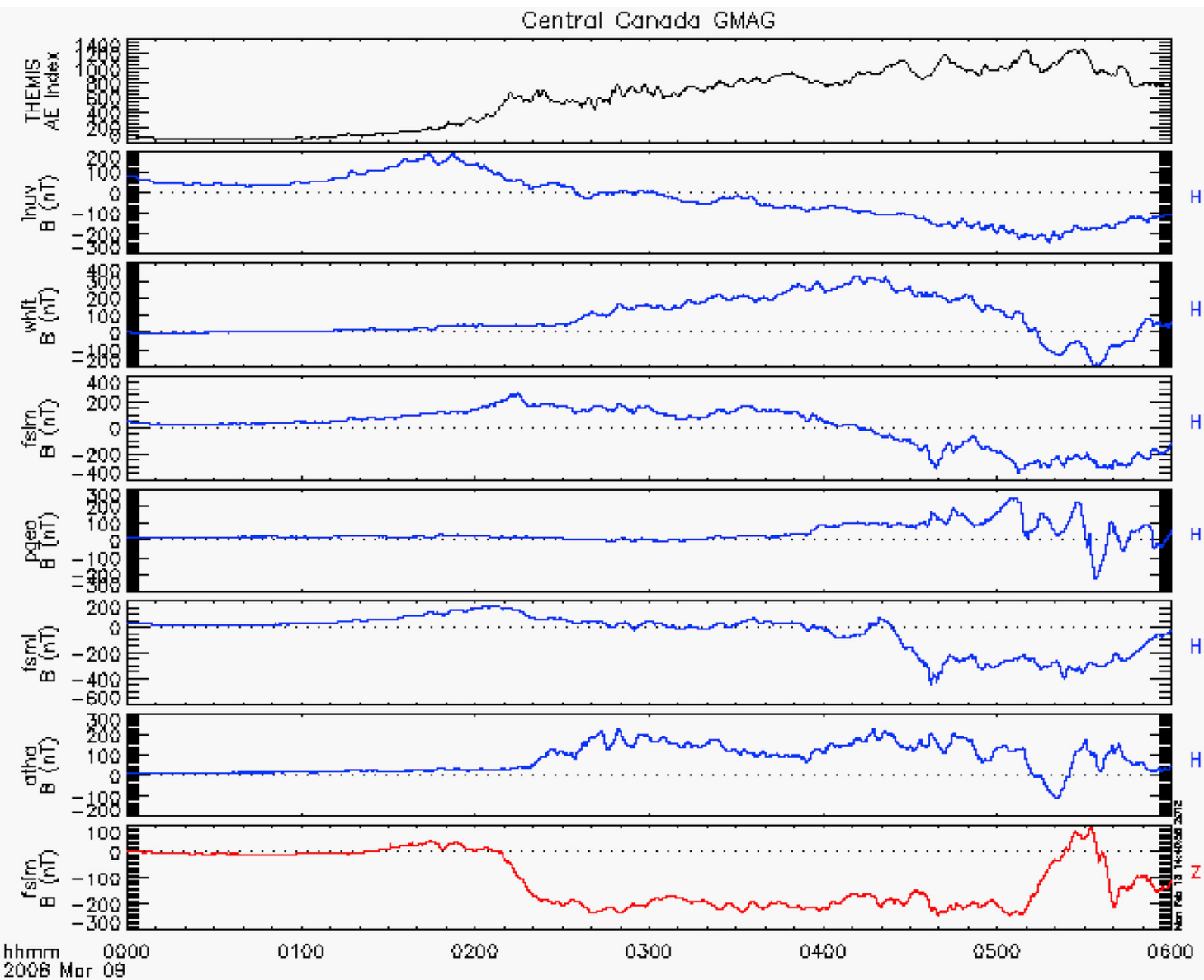

Figure 1. Horizontal ground magnetic measurements in central Canada. The first panel shows the THEMIS pseudo-AE determined from the whole network of ground magnetometers. The following traces are ordered from west to east with more poleward stations INUV, FSIM and FSMI and the more equatorward stations WHIT, PGEO, and ATHA. The eighth panel shows the vertical component of the magnetic disturbance at FSIM.

the polar cap as revealed by the FAST data, moves slowly equatorward at about $100 \mathrm{~m} / \mathrm{sec}$ during the first $7 \mathrm{~min}$ (Figure 4). This arc is a so-called Alfvénic arc, which has distinctly different features in optical appearance and electron spectrum from the other auroral arcs. Alfvénic arcs have a very pronounced coarse and short-lived ray structure, are related to a balanced current system [Mende et al., 2003], have a distributed energy spectrum of strongly field-aligned electrons and are associated with solitary electromagnetic structures of $0.1 \mathrm{~s}$ duration, with strong electric fields and magnetic fluctuations which have been interpreted as kinetic Alfvén waves [Wahlund et al., 1994]. Furthermore the Alfvénic arcs exhibit transverse ion heating and broadband ELF electrostatic noise in the topside ionosphere [Lundin et al., 1994; Wahlund et al., 1998; Stasiewicz et al., 2000]. Additionally, there are new arcs appearing at the poleward edge of the oval (long track in Figure 4) which then move equatorward with a much faster speed, on average with $300 \mathrm{~m} / \mathrm{sec}$. The new arcs almost provide the impression as if they were "born" out of the Alfvénic arc. After having become bright enough to be recognized they move equatorward. The latitude variations of the Alfvénic arc and some of the other arcs within the FOV of FSIM are given in Figure 4 . The steepness of the tracks of the other arcs shows how much faster they move equatorward than the much more stable Alfvénic arc at the poleward boundary of the oval. A similar observation, except for the presence of an Alfvénic arc, was made by de la Beaujardière et al. [1994] in the midnight sector during a quiet period. A new arc appeared poleward from an existing arc and not far from the polar cap boundary. The arcs drifted equatorward with speeds similar to the here observed ones. Such observations show that the here reported event is by no means unique. Its value lies in the long-duration optical coverage with the high temporal resolution and sensitivity of the THEMIS GBO all sky cameras. This is documented in an animation provided as auxiliary material to this paper. ${ }^{1}$

[12] One characteristic of the arcs during their equatorward motion is often a brightening and broadening followed by an unfolding. The unfolding of the arc is best seen in the movie, but in Figure 5 we try to demonstrate the unfolding in a series of still images. All-sky images taken $3 \mathrm{~s}$ apart are shown at the top and bottom and the middle represents the difference image. White areas receive more light, either because of brightening of pre-existing aurora, or through the

\footnotetext{
${ }^{1}$ Auxiliary materials are available in the HTML. doi:10.1029/ 2012JA018128.
} 

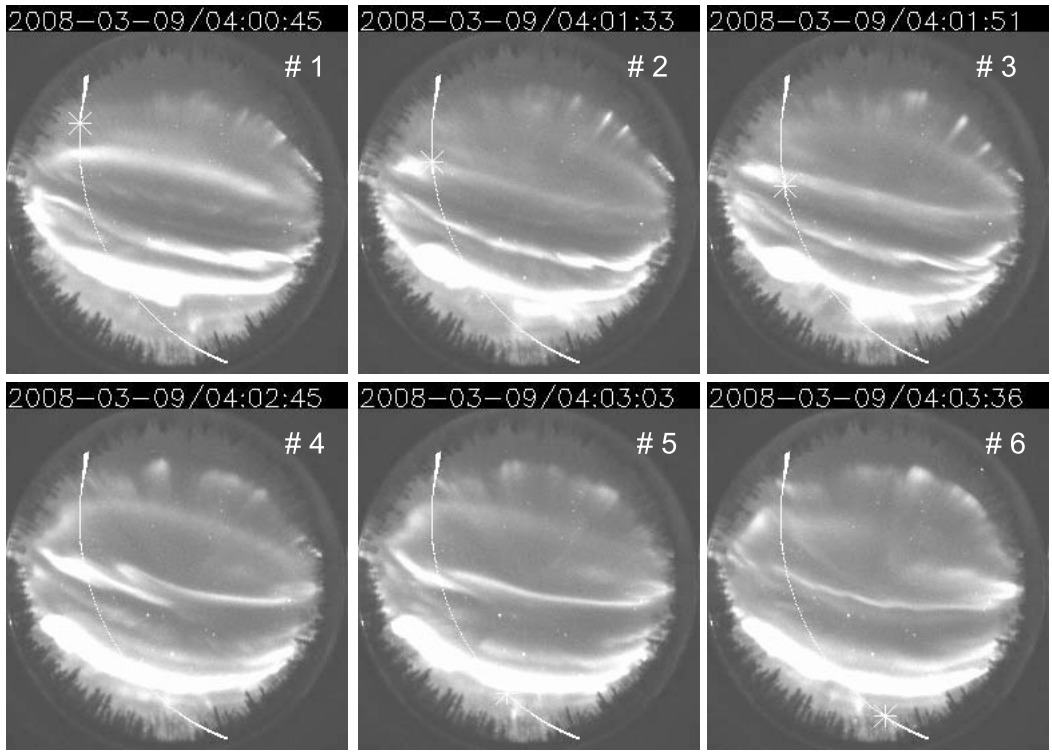

Figure 2. Summary of FSIM all-sky camera images during the crossing of FAST through the overhead aurora at the times of magnetic conjugacy with six selected arcs. The FAST location was mapped down along the magnetic field to $120 \mathrm{~km}$ and the track and instantaneous positions are given in each frame. The images are shown as if one would look down from space and therefore north is at the top and east is to the right.

motion of bright structures into a previously darker area. Black regions in the difference images become darker over the three seconds either because the aurora became weaker, or the auroral structure moved away from that region, or both. Best recognizable are the black areas directly poleward and adjacent to white structures. They signal equatorward progression of the arc.

\subsection{SuperDARN Observations}

[13] The Prince George SuperDARN HF coherent scatter radar operated by Saskatchewan University of Canada is located at geographic coordinates $\left(54^{\circ} \mathrm{N}, 122.6^{\circ} \mathrm{W}\right)$ in British Columbia. The radar operates in the frequency range from 8 to $20 \mathrm{MHz}$. The transmitted waves can be scattered at heights between 100 and $400 \mathrm{~km}$ from field-aligned density

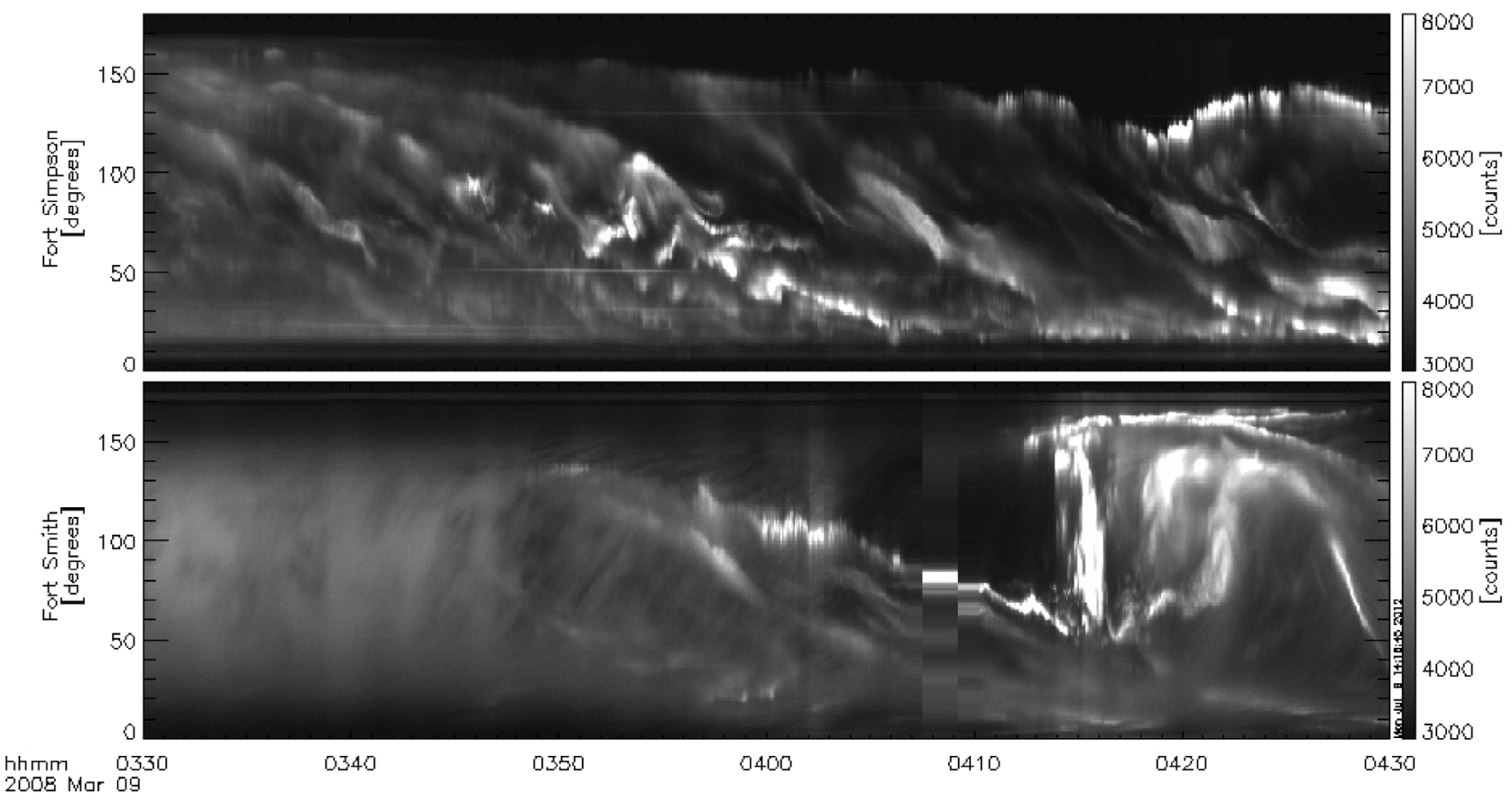

Figure 3. All-sky keograms, i.e., scans along the local magnetic north-south meridian, for stations Ft. Simpson (FSIM) and Ft Smith (FSMI). They document the overall auroral situation for the region under investigation between 03:30 UT and 04:30 UT and the continuous equatorward movements of the arcs. 


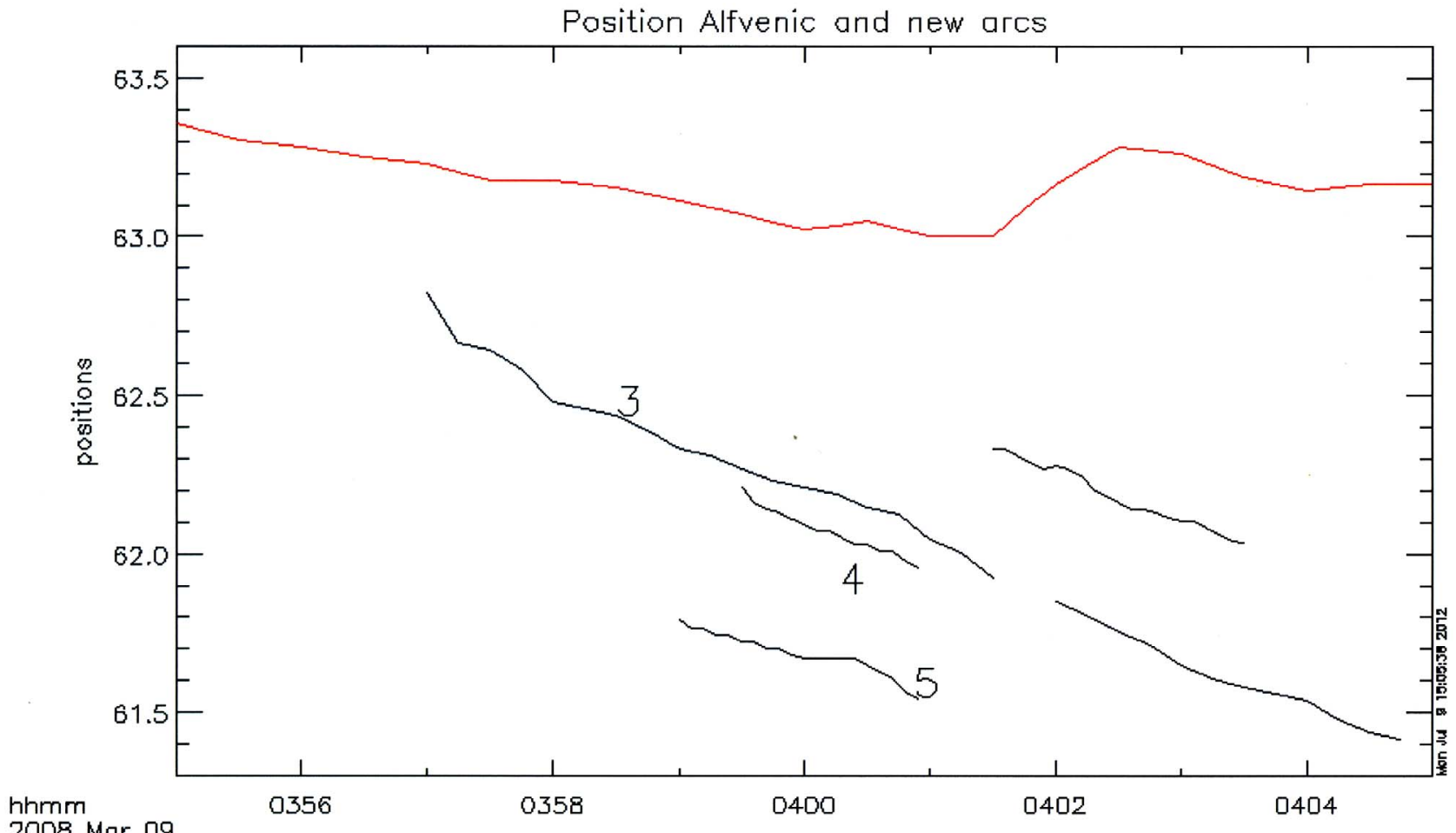

Figure 4. Magnetic latitude versus time of the Alfvénic arc (top track) and of arcs 3, 4, and 5. The newly appearing arc (long track) merged with arc 3 at the time of FAST crossing (04:01:50 UT). The arc with the track starting at 04:01:30 UT was another "new-born" arc. The speed of the Alfvénic arc is about $100 \mathrm{~m} / \mathrm{s}$ during the first $7 \mathrm{~min}$ with an average speed of $33 \mathrm{~m} / \mathrm{sec}$ for the whole $10 \mathrm{~min}$ interval, while the new arc moves at $320 \mathrm{~m} / \mathrm{sec}$. The mean speed of the three other arcs is $296 \mathrm{~m} / \mathrm{s}$. (04:00 UT corresponds to 19:02 MLT at the meridian of FSIM).

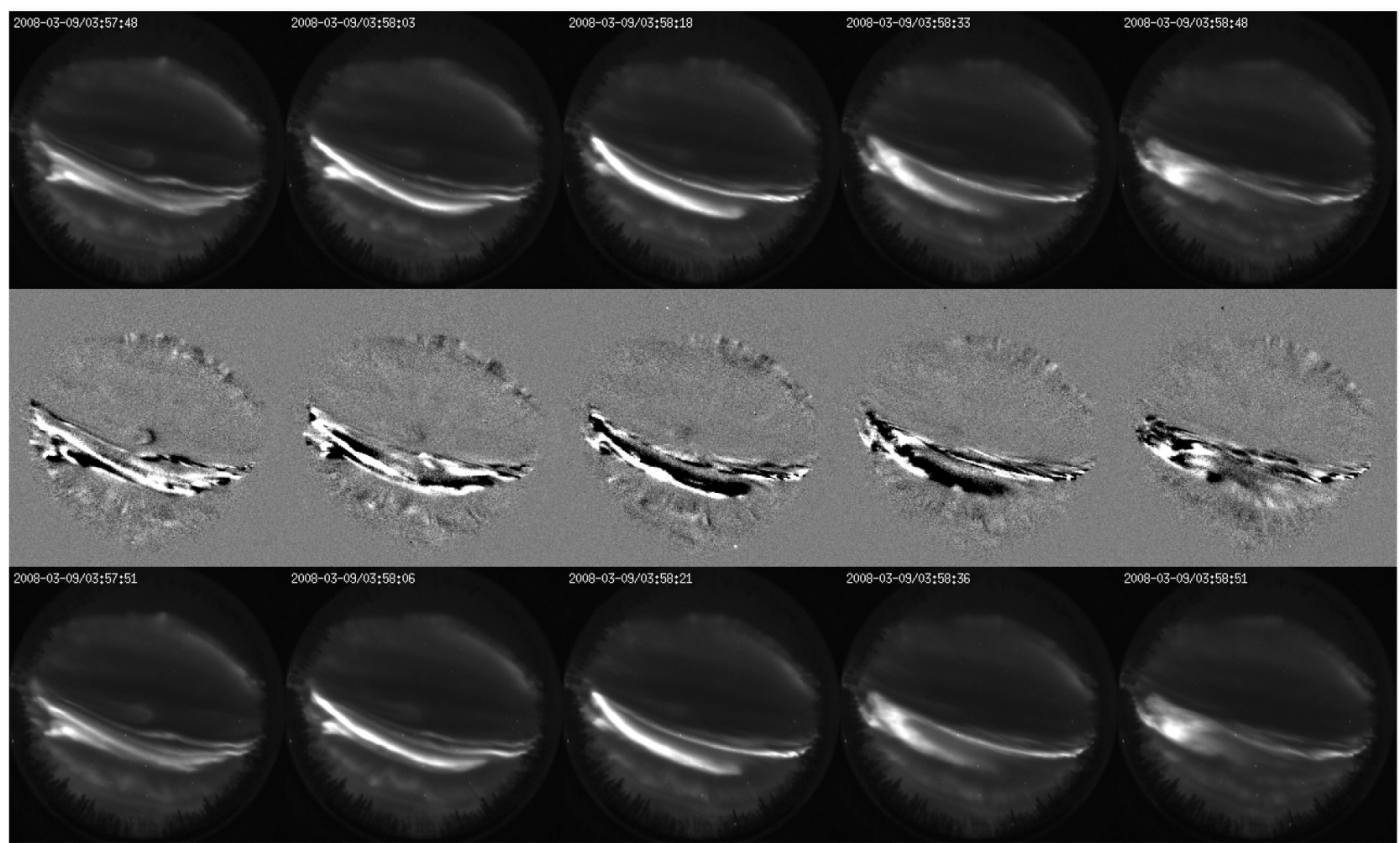

Figure 5. All sky images taken $3 \mathrm{~s}$ apart (top and bottom) with difference images in the middle. 


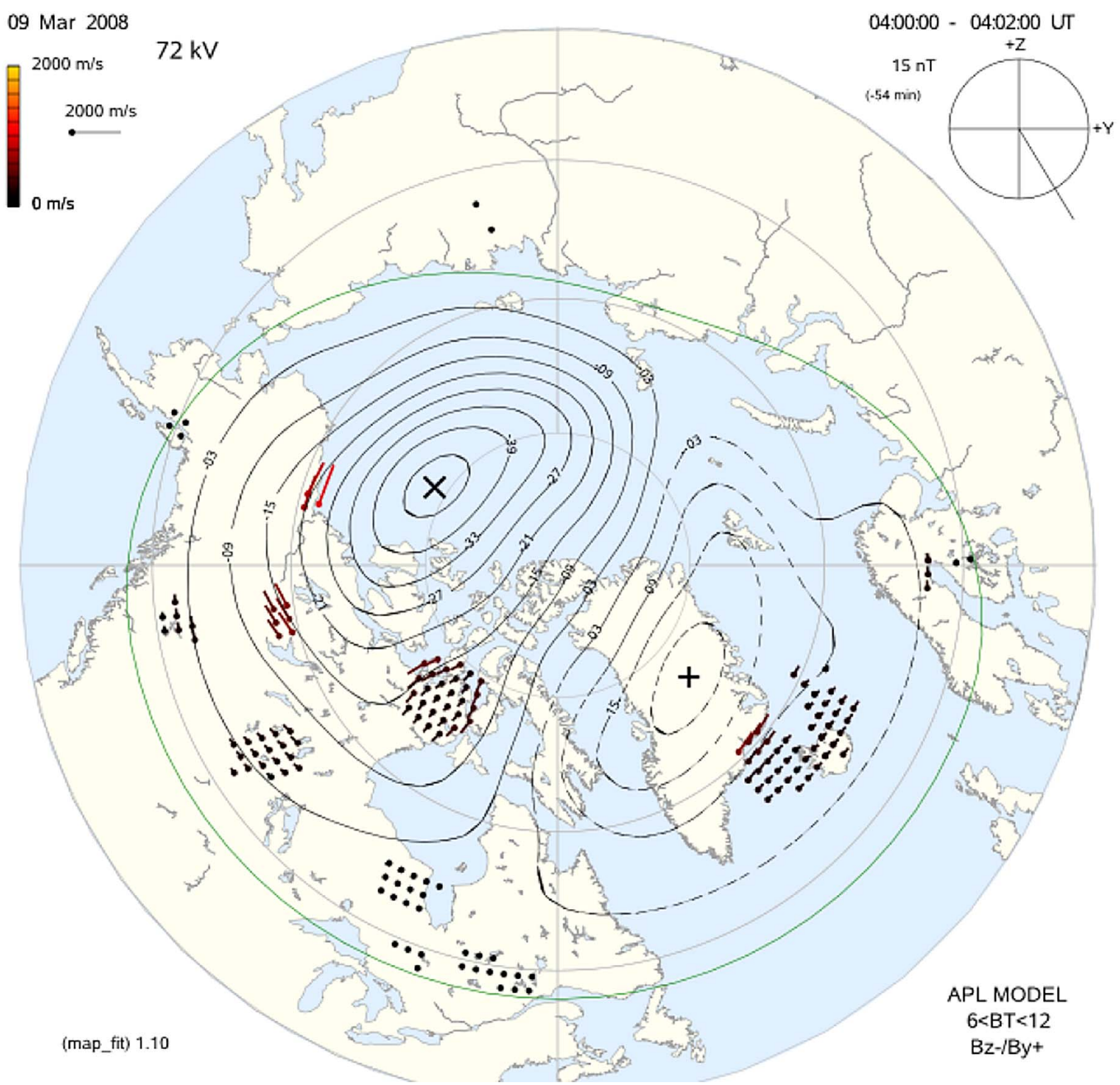

Figure 6. Composite of several fields of plasma flows derived from the SuperDARN network for 09 March 2008 and 04:00-04:02 UT. The lower red arrows coincide approximately with the FOV of FSIM and show a westward flow of about $1 \mathrm{~km} / \mathrm{s}$.

perturbations. The received signal is gated into 75 range gates along 16 beams. Each range gate covers approximately $45 \mathrm{~km}$ in the horizontal plane at the altitude of the wave scattering and the angular separation between adjacent beam directions is $3^{\circ}$. The parameters deduced from the autocorrelation function of the received signal are the velocity of electron irregularities along the radar beam, the scattered power and the spectral width. The temporal resolution in survey mode is around $2 \mathrm{~min}$.

[14] Figure 6 shows a composite of several fields of plasma flows derived from the SuperDARN network. Clearly visible is a westward oriented flow of a little more than $1 \mathrm{~km} / \mathrm{s}$ in the area covered by the FOV of the FSIM allsky camera. This flow is consistent with the westward motions of structures inside the auroral arcs and the eastward overhead current. Weygand et al. [2012] have shown that equivalent currents are nearly antiparallel to the flows. However, the magnetometer data shown in Figure 1 clearly reveal that most of the ionospheric current was concentrated south of FSIM, while the radar signals appear to be dominantly backscattered from field-aligned irregularities related to the arcs and the associated upward currents.

[15] Figure 7 shows power, line-of-sight velocity, and spectral width of the backscatter signal from the SuperDARN station at Prince George, British Columbia (Canada). A clear equatorward motion is exhibited between 03:20 and 04:10 UT by all three signals. Since the flow direction is almost precisely at right angles to the line-of-sight from Prince George, the Doppler signal captures essentially the southward propagation of the irregularities, from which the 


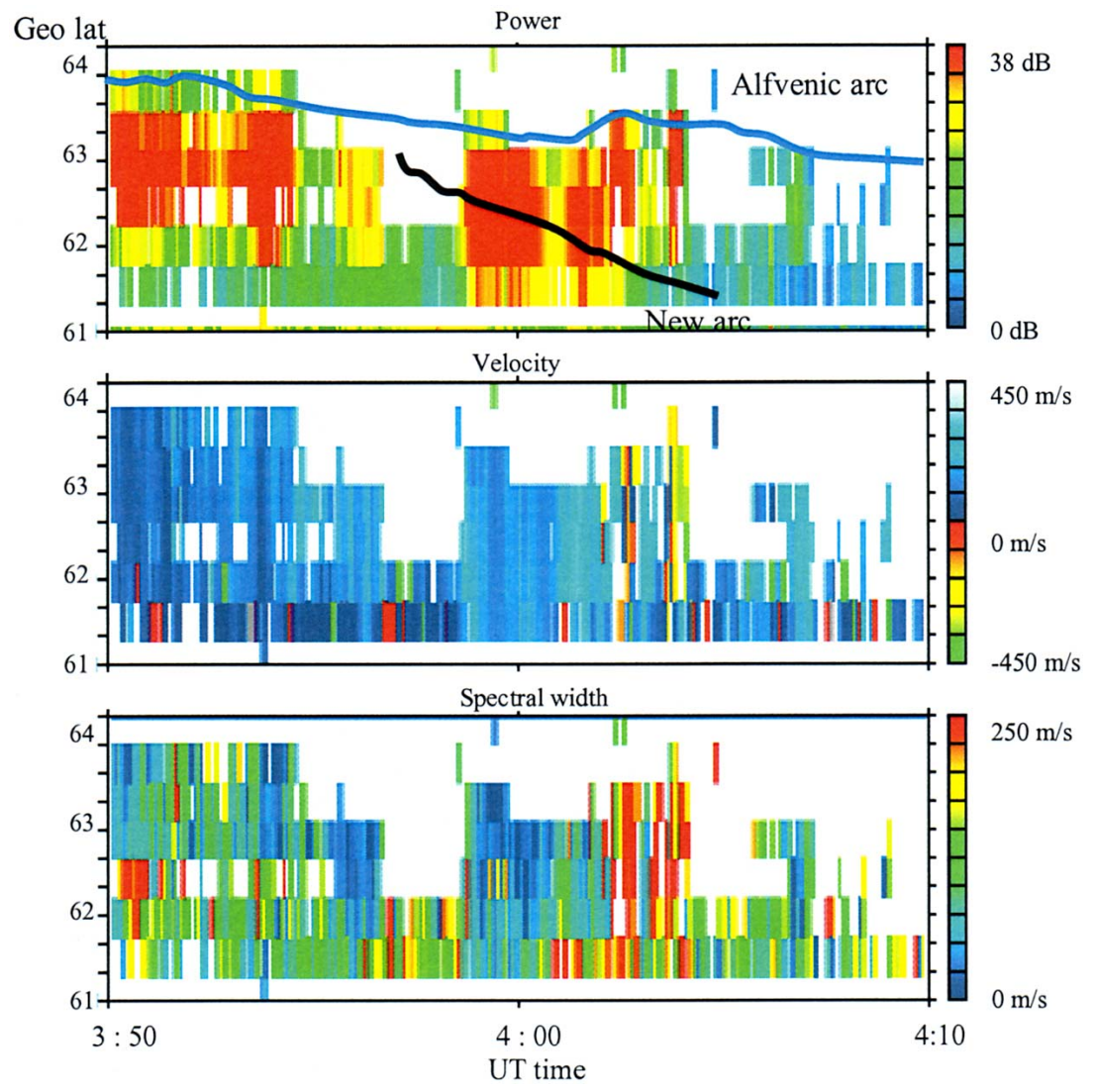

Figure 7. (top) Power, (middle) line-of sight velocity, and (bottom) spectral width of the backscatter signal from SuperDARN measurements at Prince George, British Columbia. The equatorward moving signals between 03:50 and 04:10 UT in the geographic latitude range from 66 to 61 degrees originate from the arcs under study as indicated by the black trace in Figure 7 (top). The blue trace shows the position of the Alfvénic arc.

radar signals are being scattered. The strongest power signals around 04:00 UT (Figure 7, top) correspond to the positions of the strongest arcs a bit south of FSIM. The related Doppler signals (Figure 7, middle) range between 0 and $200 \mathrm{~m} / \mathrm{s}$. It is important for the subsequent analysis to realize that the Doppler shift reveals the motion of irregularities embedded in the $\mathrm{F}$ region thus exhibiting the plasma flow. They need not necessarily agree with the optically observed propagation velocity of the arcs, which may be a sum of proper motion relative to the plasma frame and the motion of that frame [Haerendel et al., 1993; Frey et al., 1996; del Pozo et al., 2002]. It may be noted in passing that no signals have been obtained from the Alfvénic arc.

\subsection{Equivalent Currents}

[16] In order to obtain the ionospheric equivalent currents for this event, data from more than 60 magnetometer stations have been analyzed with the Spherical Elementary Current Systems (SECS) technique [Amm, 1997; Amm and Viljanen, 1999]. The SECS technique makes use of the fact that the ionospheric (and ground) equivalent currents are always divergence-free [e.g., Fukushima, 1976]. Therefore they can be expressed as a superposition of divergence-free current systems. The locations of the SECS poles are distributed over the region of interest, and each SECS pole may have different amplitude. The magnetic field of the SECS can be expressed analytically. This allows calculating the ionospheric equivalent current density by determining the amplitudes of the SECS poles such that the superposed ground magnetic effect of the SECSs optimally matches the measured disturbance magnetic field on the ground. The locations of the SECS poles can be chosen freely, in order to best accommodate the location and density of the available measurement points. For the combined magnetometer network used here, the spatial resolution of the resulting ionospheric equivalent currents is $300 \mathrm{~km}$. The vertical component of the curl of the ionospheric equivalent currents, which can directly be derived from the amplitudes of the SECS poles, is proportional to the field-aligned current density (FAC) in the case that the conductance is uniform, or in case that conductance gradients point along the electric field direction [Amm et al., 2002].

[17] Figure 8 shows the result of this analysis for 04:00 UT. In the area covered by the FOV of FSIM (left circle centered at $-120^{\circ}$ longitude) there is a clear eastward electrojet ranging between 300 and $700 \mathrm{~A} / \mathrm{km}$. The distribution of blue and red colors, which are indicative for upward and downward field-aligned currents, respectively, show that the first are co-spatial with the auroral arcs. The strongest horizontal currents are found at the transition from up- to downward 


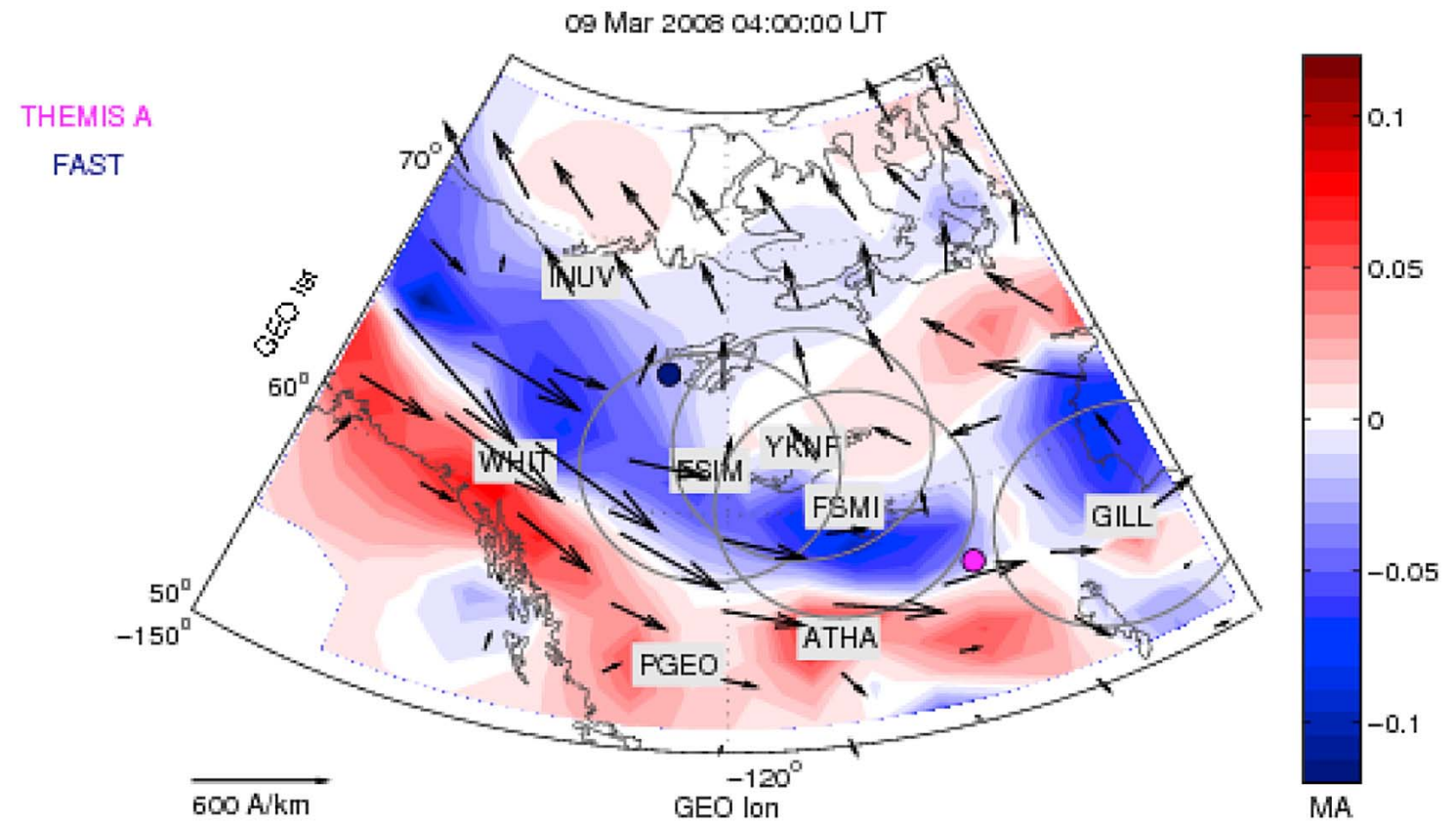

Figure 8. Equivalent currents for the Canadian sector at 04:00 UT. Black arrows show current direction and strength. Red and blue colors show areas of downward and upward field-aligned currents, respectively. Colored circles indicate the foot points of the FAST and THEMIS-A spacecraft.

currents, where the horizontal electric field and thus the Hall current is expected to maximize.

[18] The map shown in Figure 8 contains in addition the positions of the magnetic foot points of the five THEMIS spacecraft calculated with the Tsyganenko [1989] model for $\mathrm{Kp}=3$. Note in particular that of THEMIS-A (red circle) which is closest to the area of investigation.

\subsection{The THEMIS Spacecraft}

[19] The five identical satellites of the THEMIS mission [Angelopoulos, 2008] allow tracking the motion of plasma and waves from one point to another and resolve space-time ambiguities in key regions of the magnetosphere. The probes are equipped with comprehensive and identical particles and fields instruments. During this event the THEMIS-A spacecraft was located a little more than one hour east from the meridian plain through FSIM at a geocentric distance of 7.6 $\mathrm{R}_{\mathrm{E}}$ in a high-beta environment. Judging from the position of the foot point of the spacecraft (Figure 8) well inside the FOV of the neighboring station, FSMI, and slightly magnetically south of FSIM, one may rightly conclude that the plasma and field data measured by THEMIS-A are likely to be representative for the source region feeding the auroral arcs.

[20] As shown by Figure 9, the particle and field data were nearly constant during the time interval relevant to this investigation, except for the z-component of the electric field, which changed from positive to slightly negative during the first three minutes and then was essentially zero. The initial northward component of $\mathrm{E}_{\mathrm{z}}$ is consistent with the westward motions in the FSIM area. Most important for our subsequent analysis are the electron density and temperature, and the plasma beta, which were about $1.3 \mathrm{~cm}^{-3}, 2.5 \mathrm{MK}$, and near 2.0 , respectively.

\subsection{The FAST Measurements}

[21] The FAST spacecraft has been described in detail by Carlson et al. [1998]. We will only remark that at the time of the event the dc electric field measurements were no longer available. Figure 10 shows energy-time spectra of electrons and ions and their angular distributions together with the transverse magnetic component and the downward electron energy fluxes projected into the ionosphere for $8 \mathrm{~min}$ after 03:59:00 UT on 09 March 2008. FAST crosses the FSIM area at an altitude of 3300 to $3500 \mathrm{~km}$ and is essentially below the acceleration region. Six intervals with inverted-Vtype electron spectra corresponding to auroral arcs have been selected for evaluation. All six can be identified in the all sky images. Arc 1 is a newborn one and still very weak. FAST crossed its current sheet where a wide fold was developing. This explains the large interval of contact. Arcs 2 and 3 actually belong to the same arc which was in the process of splitting where FAST was crossing. The arc expressed by a distributed energy spectrum and dominantly downward directed fluxes shortly after 03:59:30 UT is the Alfvénic arc at the polar border of the auroral oval. This arc behaved very differently from the other arcs, as evident from the keogram and the latitudinal motion shown in Figure 4. We will return to this circumstance below.

[22] All six selected arcs appear in upward field-aligned currents, as indicated by the positive, in some cases clearly enhanced, upward slopes of the $B_{\perp}$ contour. There are two intervals with downward currents, the first one following immediately arc 6, the second one after 04:06:15 UT, when the foot point of FAST were already outside the FOV of FSIM. It is curious that there is a clear (though weak in energy flux) signature of a further arc from 04:04:21 to 04:04:51 UT, unfortunately already hidden outside the border of the all sky images, for which $B_{\perp}$ is mostly decreasing, 


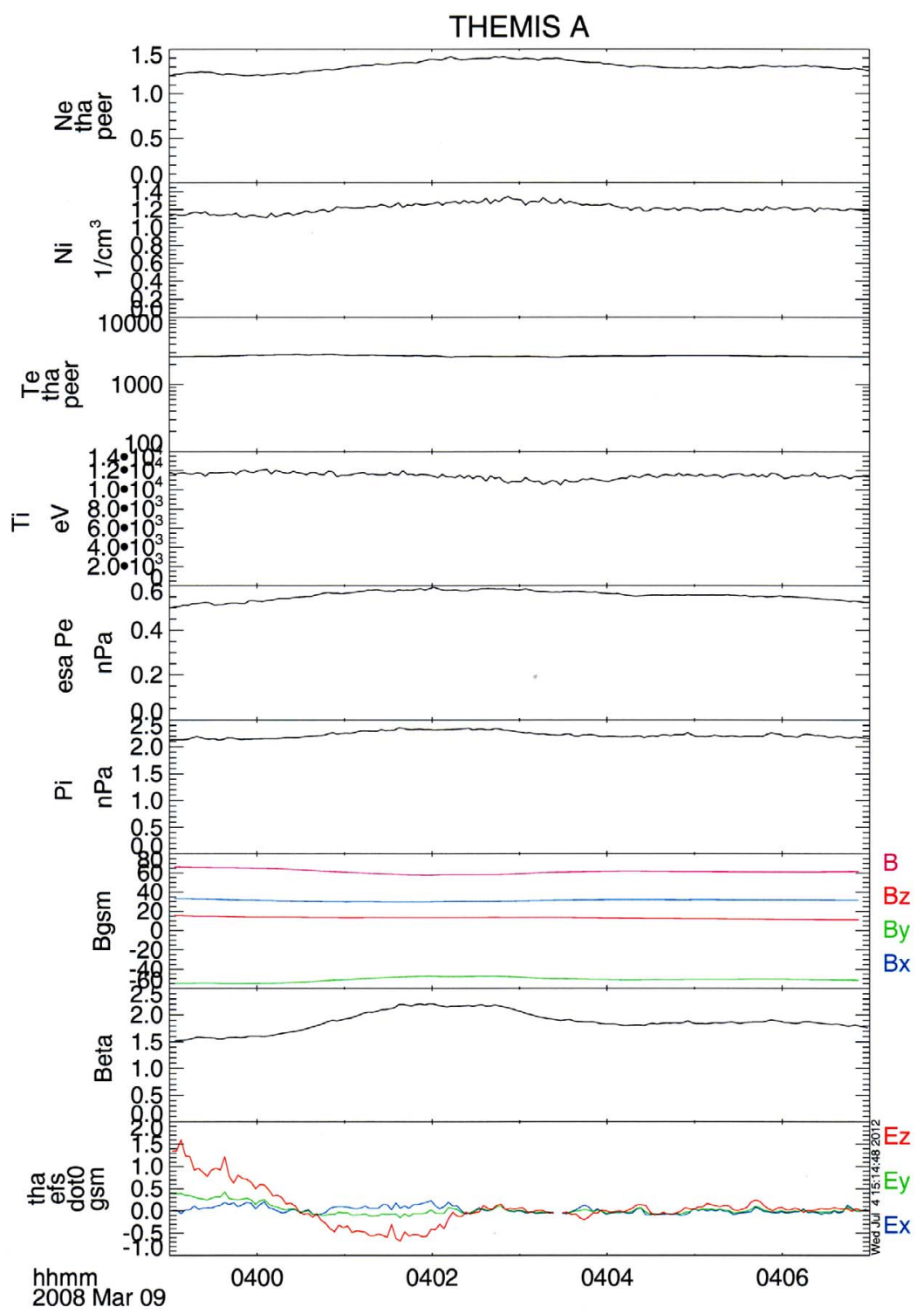

Figure 9. THEMIS A data for eight minutes covering the period under study. From top to bottom the panels show: electron density, ion density, electron temperature, ion temperature, electron pressure, ion pressure, total field strength and components in GSM coordinates, plasma beta, and electric field components in GSM coordinates. The position of THEMIS-A at 04:00 UT was at $r=7.58 R_{E}, z=-2.6 R_{E}$, at 20:40 MLT.

indicating downward currents. Furthermore, we point out that the $B_{\perp}$ contour does not return to its initial level at 04:00:00 UT giving the impression of an unbalanced fieldaligned current system. Unfortunately the FAST data interval ends before having left the equatorward section of the oval dominated by downward currents. However, there is no necessity for such a balance, as observed by Fujii et al. [1994] and shown by Marghitu et al. [2004].

\section{Data Reduction and Comparison With Theory}

[23] Before addressing the observed arc motions we will try to interpret the quantities measured by FAST in the framework of a theory by Haerendel, [2007] which treats auroral arcs embedded in a convective flow along the auroral oval. The model is based on a large-scale current configuration of Type II following the nomenclature of Boström [1964], in which upward currents on the poleward side of the oval are balanced by the downward currents on the equatorward side. The auroral arc was thought to be produced inside a narrow sheet of high current density but containing only a small fraction of the total upward current. Although strict balancing does not seem to exist in our event, at least the condition of small sheet currents through the arcs is fulfilled. The main characteristic of this model, distinguishing it from other approaches, is that the arcs are energized by releasing magnetic shear stresses by means of the decoupling action of the potential drops along the acceleration region. The shear stresses are set up by the large-scale 


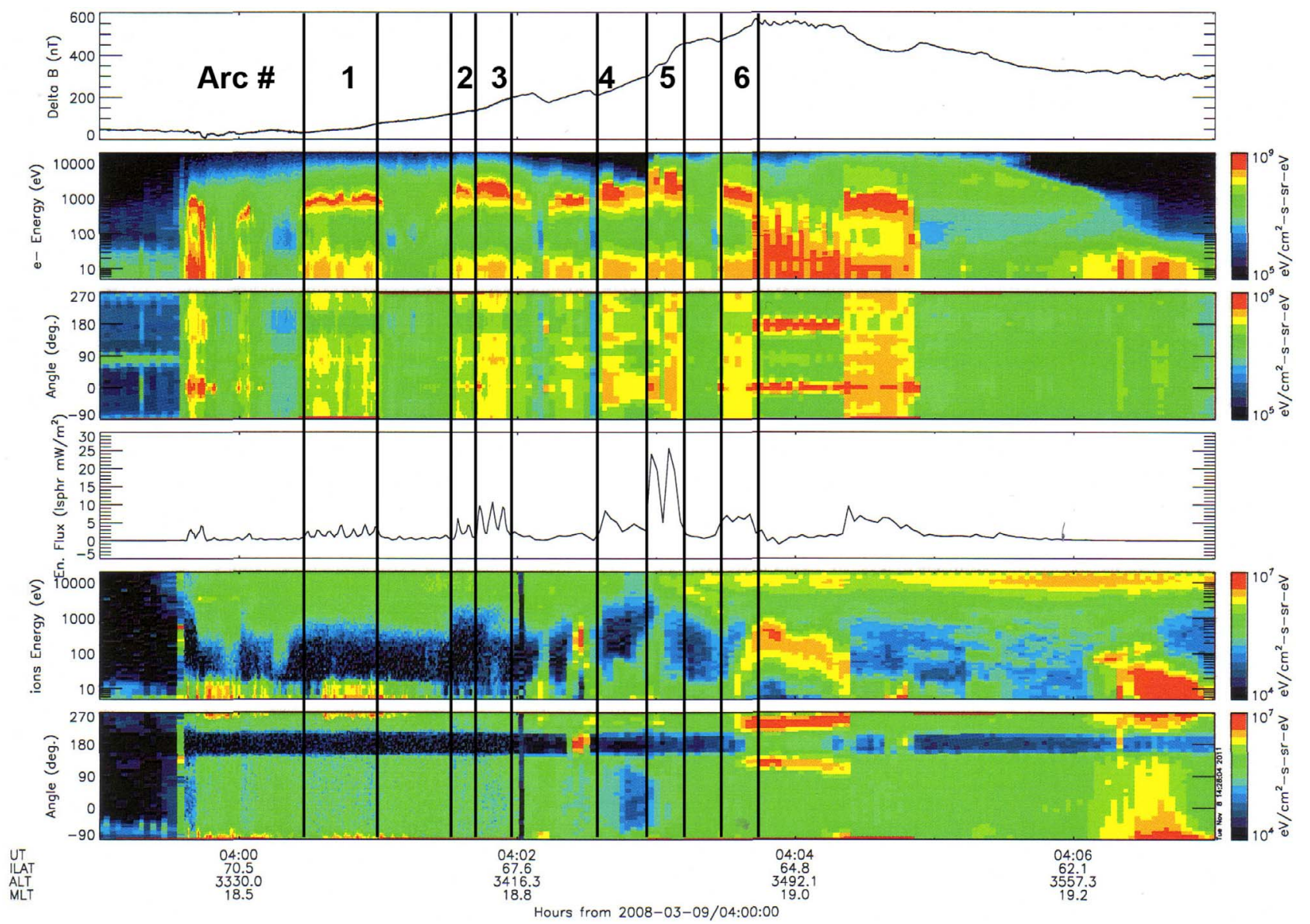

Figure 10. FAST data for the crossing of the FOV of FSIM. (first panel) Magnetic component perpendicular to the undisturbed (model) field; (second and third panels) electron energy-time spectra and pitch angle distributions; (fourth panel) energy flux projected to ionospheric height; (fifth and sixth panels) ion energy-time spectra and pitch angle distributions. The six analyzed arcs are numbered. They exhibit clear inverted-V signatures, in contrast to the Alfvénic arc at 03:59:30 UT which shows a strongly field-aligned velocity distribution.

current system and assumed to be maintained independently from the presence of an arc. Consequently, the arc propagates into the interior of the gross current system while releasing the shear stresses. This proper motion occurs relative to the plasma frame. The great promise of the data set analyzed here is that it offers the potential of providing an intricate consistency check of the theory, by comparing observed arc motions with the theoretical proper motions. An important simplification of the theory is to use only quantities integrated or averaged across the auroral current sheet. Thus one cannot expect precision but only plausibility and internal consistency of the whole set of represented auroral parameters. In order to facilitate the reference to the theoretical framework, we make use of the formalism laid down in a recent paper of the first author [Haerendel, 2012] especially conceived for such purpose.

\subsection{Data From the FAST Spacecraft}

[24] Figure 11 depicts the situation. We derive the sheet current density through the arc by the readings of $B_{\perp}$ on either side of the current sheet (subscript ' $u$ ' for upstream and ' $d$ ' for downstream along the arc's motion) and project it to the ionosphere. This is key information for the energy input into the arc and ionosphere. The conductivity is enhanced by the electron precipitation and is attached to the frame of the neutral constituent which is assumed to be at rest in the observer's frame. The ambient electric field tangential to the arc, $E_{t}$, drives a N-S Hall current thus creating the situation for Cowling conductivity. In the frame of the conductivity enhancement, $E_{t}$ is westward directed. Besides the data obtained from the FAST mission we will make use of the empirical relations for the Pedersen and Hall conductivities as functions of the electron energy flux and mean energy by Robinson et al. [1987].

[25] The FAST data provide the following quantities: $\dot{W}_{\text {arc }}$, $\Phi_{\|}, B_{\perp u}, B_{\perp d}$, and $\mathrm{w}_{\text {arc }} . \Phi_{\|}$is actually not the potential drop but $\bar{E}_{e l} / e$, where $\bar{E}_{e l}$ is the average energy of the electrons. $\mathrm{w}_{\mathrm{arc}}$ is the width of the arc projected to the ionosphere and corrected for its motion in the direction of the spacecraft trajectory. The energy flux, $\dot{W}_{\text {arc }}$, has already been transformed to its value at ionospheric level, whereas the transverse magnetic field data of Figure 10 apply to the satellite 


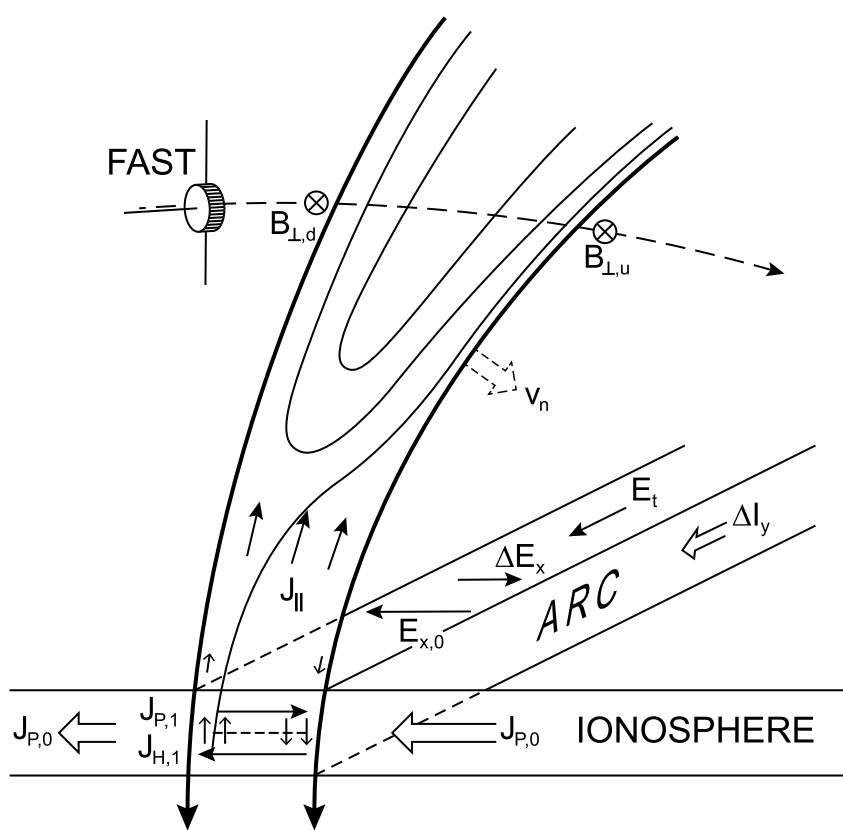

Figure 11. Configuration of one of the arcs overflown by FAST and the associated currents and electric fields. $\mathrm{J}_{\mathrm{H}, 1}$ and $\mathrm{J}_{\mathrm{P}, 1}$ are the Hall and Pedersen currents created inside the conductivity enhancements by the tangential electric field, $E_{t}$. An excess Hall current contributes to the fieldaligned currents (short vertical arrows). $\mathrm{E}_{\mathrm{x}, 0}$ is the electric field associated with the primary Pedersen current, $\Delta \mathrm{E}_{\mathrm{x}}$ and $\Delta \mathrm{I}_{\mathrm{y}}$ are the perturbations of that field and of the total eastward current along the arc. $v_{n}$ indicates the equatorward proper motion of the arcs.

altitude. Multiplication by a 1.87 , the mapping factor for sheet currents between spacecraft and $\mathrm{E}$ region, yields the magnitude at the ionosphere. From the latter we derive the field-aligned sheet current density, $J_{\|}$, emerging from the ionosphere and the Pedersen current, $J_{P}$, by:

$$
\begin{gathered}
J_{\|}=\frac{1}{\mu_{0}}\left(B_{\perp u}-B_{\perp d}\right) \\
J_{P}=\frac{B_{\perp u}+B_{\perp d}}{2 \mu_{0}} .
\end{gathered}
$$

The total rate of energy available for conversion into auroral particle energy and ionospheric dissipation is derived from the magnetic stress release and according to Haerendel [2012] can be expressed by:

$$
\dot{W}_{\text {tot }}=\frac{B_{\perp u}^{2}-B_{\perp d}^{2}}{2 \mu_{0}} \cdot \frac{R_{w}}{\mu_{0}} .
$$

Magnetic stress release is a complex dynamic process involving feedback to and from the generator plasma [Haerendel, 2007, section 7]. Whatever the latter contribution is, it will be contained in the difference between the magnetic energy contents in front and behind the arc. It is assumed that all energy is transported in the Alfvén wave mode along the magnetic field lines. This is manifested by $R_{w}$, the associated wave impedance. In the quasi-stationary situation underlying the theory, $R_{w}$ is defined as:

$$
R_{w}=\frac{\mu_{0} \ell_{\text {eff }}}{\tau_{\text {arc }}}=\frac{2 \mu_{0} R_{E} L}{\tau_{\text {arc }} \Gamma^{2}} .
$$

The term $\ell_{\text {eff }}=2 R_{E} L / \Gamma^{2}$ is the effective length of an auroral flux tube between ionosphere and generator. $R_{E}$ is the Earth's radius and $\mathrm{L}$ the magnetic shell parameter. $\tau_{\text {arc }}$ is the effective interaction time between the arc and the generator plasma. Haerendel [1994, 2007] estimated that this time would amount to about four Alfvénic transit times, $\tau_{A}$, between high latitude ionosphere and equator. $\Gamma^{2}$ is a numerical factor that stems from the distorted dipole model employed in the theory of Haerendel [2007]. It depends on the plasma beta at the equator, $\beta_{e q}$, and ranges between 1.1 and 1.35. It is contained in [Haerendel, 2012, Figure 2]. While $R_{w}$ will be derived later, the factor in front of it in equation (3) can be obtained directly from the satellite data.

[26] Table 1 contains the directly obtained satellite data and quantities derived from equations (1)-(3). The only monotonically varying quantities in this table are the Pedersen current and, except for arc 6 , the differential magnetic energy density. The latter reflects the increasing tangential stresses of the magnetic field toward the center of the current system. All arcs, except for the curious one encountered by FAST after 04:04:21 UT (Figure 10), are located on the poleward side of the maximum of the Pedersen current. In a simple Type II current geometry, this is the region of generally upward directed currents. The arc widths derived from the timing of the auroral electron fluxes were corrected for the equatorward motion of the arcs which was measured to be close to $300 \mathrm{~m} / \mathrm{s}$. In the subsequent data evaluations, we will not make use of the observed arc widths except for comparing them with those derived by means of our theoretical tools.

\begin{tabular}{|c|c|c|c|c|c|c|c|c|}
\hline Arc & $B_{\perp u}(\mathrm{nT})$ & $B_{\perp d}(\mathrm{nT})$ & $J_{\|}(\mathrm{A} / \mathrm{m})$ & $J_{P}(\mathrm{~A} / \mathrm{m})$ & $\left(B_{\perp, u}^{2}-B_{\perp, d}^{2}\right) / 2 \mu_{0}\left(\mathrm{erg} / \mathrm{cm}^{3}\right)$ & $\dot{W}_{\text {arc }}\left(\mathrm{mW} / \mathrm{m}^{2}\right)$ & $\Phi_{\|}(\mathrm{kV})$ & $\mathrm{w}_{\mathrm{arc}}(\mathrm{km})$ \\
\hline 1 & 131.5 & 0 & 0.1046 & 0.0523 & $6.880 \times 10^{-8}$ & 2.01 & 1.402 & 77.0 \\
\hline 2 & 236.7 & 171.9 & 0.0516 & 0.1626 & $1.054 \times 10^{-7}$ & 2.32 & 1.676 & 24.0 \\
\hline 3 & 394.0 & 242.0 & 0.1210 & 0.2530 & $3.846 \times 10^{-7}$ & 7.60 & 2.547 & 37.3 \\
\hline 4 & 546.4 & 394.6 & 0.1208 & 0.3744 & $5.682 \times 10^{-7}$ & 4.54 & 1.770 & 51.3 \\
\hline 5 & 837.2 & 562.3 & 0.2188 & 0.5518 & $1.531 \times 10^{-6}$ & 21.1 & 3.717 & 38.4 \\
\hline 6 & 1051.1 & 889.0 & 0.1289 & 0.7719 & $1.251 \times 10^{-6}$ & 7.62 & 2.190 & 37.9 \\
\hline
\end{tabular}

Table 1. Transverse Magnetic Field Components on Either Side of an Auroral Current Sheet, Field-Aligned Sheet Current Density, Mean Height-Integrated Pedersen Current Within the Arc, Differential Magnetic Energy Density, Measured Electron Energy Flux, Parallel Potential Drop, and Measured Arc Width, for Six Arcs Traversed by FAST on 09 March $2008^{\mathrm{a}}$

${ }^{\mathrm{a}}$ All quantities are referring to ionospheric height. 


\subsection{Derivation of Environmental Parameters and Some Arc Properties}

[27] From the quantities assembled in Table 1 we can derive various environmental parameters and arc properties which are not directly observable or serve for comparison with the measurements. The Knight relation [Knight, 1973] leads to:

$$
\begin{gathered}
j_{\|}=\frac{\dot{W}_{\text {arc }}}{\Phi_{\|}} \\
K=\frac{\dot{W}_{\text {arc }}}{\Phi_{\|}^{2}} .
\end{gathered}
$$

As $\dot{W}_{a r c}$ is an average across the current sheet, also $j_{\|}$has the meaning of an average. It serves here only as an indicator of the strength of the field-aligned current and of the applicability of the Knight relation. $K$ is the conductance appearing in the quasi-Ohm's law of the Knight relation. This relation with the above definition of the average $j_{\|}$leads to an expression for the current width:

$$
\mathrm{w}_{\text {arc }}=\frac{\left(B_{\perp u}-B_{\perp d}\right) \cdot \Phi_{\|}}{\mu_{0} \dot{W}_{\text {arc }}} .
$$

The arc width, $\mathrm{w}_{\text {arc }}$, will be later compared with the measured values and $K$ with the theoretical expression. All these expressions imply that we neglect the energy of the source electrons, $T_{e \|}$, against the average energy, $\vec{E}_{e l}=e \Phi_{\|}$, of the post-accelerated electrons [see Haerendel, 2012, equations 35-37].

[28] The total flux of liberated energy, $\dot{W}_{t o t}$, (equation (3)) is shared between the energy conversion in the auroral acceleration region and the dissipation in the ionosphere. With the knowledge of $\dot{W}_{\text {arc }}$ and $\Phi_{\|}$we can derive empirical expressions for the conductivities generated by the precipitating electrons, at least if the latter are sufficiently strong to dominate the background ionization. Besides the Pedersen current derived from equation (2), we must also account for the presence of a Hall current in case of a finite tangential electric field, $E_{t}$, and the secondary Pedersen current which partially balances the transverse Hall current, $\mathrm{J}_{\mathrm{H}, 1}$ (see Figure 11). From Haerendel [2012] we take the following expression for the energy dissipation by the two contributions to the Pedersen current:

$$
\dot{W}_{i o n}=\frac{\left(B_{\perp u}+B_{\perp d}+2 \mu_{0} \hat{\alpha}^{-1} \Sigma_{H} E_{t}\right)^{2}}{4 \mu_{0}^{2} \Sigma_{P}}+\Sigma_{P} E_{t}^{2}
$$

The expression $\hat{\alpha}^{-1} \Sigma_{H} E_{t}$ is the secondary Pedersen current, $\mathrm{J}_{\mathrm{P}, 1}$. For $\hat{\alpha}>1$ there is an excess Hall current closing in the magnetosphere. The magnitude of $\hat{\alpha}$ has been a matter of extensive discussion, beginning with Kan et al. [1984] and Rothwell et al. [1984] and again taken up by Fujii et al. [2011]. Haerendel [2009] derived an explicit expression for $\hat{\alpha}$ by considering the closure of the excess Hall current by inertial currents in the magnetosphere:

$$
\hat{\alpha}=1+\frac{1}{R_{w} \Sigma_{P, a r c}} .
$$

Equation (9) to be employed here contains the yet to be determined integral wave impedance, $R_{w}$. This necessitates iterative solutions of $R_{w}$. $\Sigma_{\mathrm{P}}$ as well as $\Sigma_{\mathrm{H}}$, however, are found directly from the empirical relations obtained by Robinson et al. [1987]:

$$
\begin{gathered}
s=\frac{\Sigma_{H}}{\Sigma_{P}}=0.45 \cdot \tilde{\Phi}_{\|}^{0.85} \text { with } \tilde{\Phi}_{\|}=\frac{\bar{E}_{e l}}{1 \mathrm{keV}} \\
\Sigma_{P}=40 S \cdot \frac{\tilde{\Phi}_{\|}}{16+\tilde{\Phi}_{\|}^{2}} \cdot \sqrt{\frac{\dot{W}_{\text {arc }}}{1 m W / m^{2}}} .
\end{gathered}
$$

The basis for deriving $R_{w}$ is the total energy equation:

$$
\dot{W}_{\text {tot }}=\dot{W}_{\text {arc }}+\dot{W}_{i o n} .
$$

With $\dot{W}_{t o t}$ from equation (3), the measured value of $\dot{W}_{\text {arc }}$, and $\dot{W}_{i o n}$ defined by equation (8), we can solve for $R_{w}$ :

$$
\begin{aligned}
R_{w}= & \frac{\delta}{K \mathrm{w}_{a r c}^{2}}+\frac{1}{\delta \Sigma_{P}} \cdot\left[\left(1+\frac{2 \mu_{0} \hat{\alpha}^{-1} \Sigma_{H} E_{t}}{B_{\perp u}+B_{\perp d}}\right)^{2}\right. \\
& \left.+\left(\frac{\Sigma_{P} E_{t}}{J_{P}}\right)^{2}\right]
\end{aligned}
$$

with

$$
\delta=\frac{J_{\|}}{J_{P}}=2 \frac{B_{\perp u}-B_{\perp d}}{B_{\perp u}+B_{\perp d}} .
$$

We solve equation (13) iteratively by setting initially $\hat{\alpha}=1$ and then use equation (9) for obtaining $\hat{\alpha}$ and a new value for $R_{w}$, etc.

[29] The last step of the conversion of the observables into environmental quantities and arc properties is the determination of $\tau_{\text {arc }}$ from $R_{w}$ by using equation (4). Here the actual value of the magnetic shell parameter, $L$, comes into play. The simple dipole relation between arc latitude and equatorial distance of the field line does not work, since the presence of auroral arcs implies high beta of the source region. Without knowing the actual beta at the origin of the different arcs, we assume throughout $\beta_{e q}=2$, because theoretical arc models show that appreciable energy fluxes into the arc require a source beta of this order [Haerendel, 2007, 2009]. This is also consistent with the THEMIS-A data (Figure 9). We derive $\mathrm{L}$ from the invariant latitude, $\lambda_{\mathrm{arc}}$, according to the relation for the distorted dipole model of Haerendel [2007]:

$$
L\left(\beta_{e q}\right)=\frac{\left(1+\beta_{e q}\right)^{1 / 6}}{\cos ^{2} \lambda_{\text {arc }}} \cdot \frac{r}{1 R_{E}} .
$$

Before turning to the evaluation we have to determine the tangential electric field. There is a most interesting difference between the equatorward motions of the arcs and the plasma. The latter motions are revealed by the SuperDARN Doppler shifts and range around $100 \mathrm{~m} / \mathrm{s}$ (Figures 7 and 12), whereas the arcs move with a speed of about $300 \mathrm{~m} / \mathrm{s}$. Interestingly, the Alfvénic arc which borders the auroral oval appears to adhere to the plasma frame, since its average equatorward speed is also about $100 \mathrm{~m} / \mathrm{s}$ (Figure 4). However, the keograms in Figure 3 show that this motion can also turn around for a 


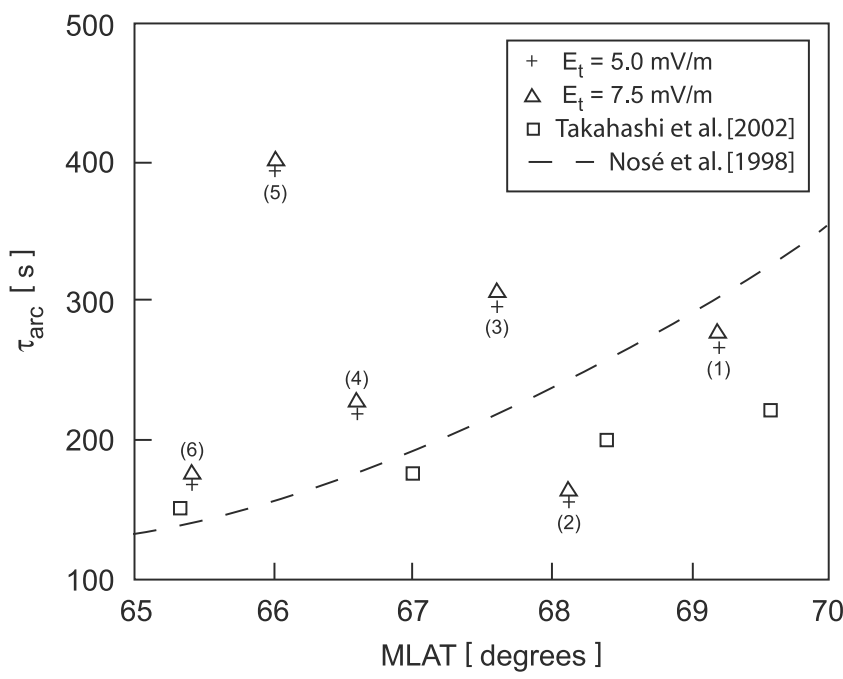

Figure 12. Derived interaction time scales, $\tau_{\text {arc }}(+$ and triangles), for six arcs, eigenperiods of fundamental toroidal standing Alfvén waves (open squares) from [Takahashi et al., 2002], and twice the fundamental periods of azimuthal Pc 4 pulsations (dashed line) calculated by Nosé et al. [1998].

short while and then resume the equatorward drift. (It should be noted that the SuperDARN measurements exhibit no sign of backscatter from the Alfvénic arc. The echoes come from the area where the brighter arcs, e.g., arcs 3-6 are located.)

[30] We interpret these findings, and will substantiate them later, as clear evidence that the arcs, except for the Alfvénic one, have a proper motion relative to the plasma frame of about $200 \mathrm{~m} / \mathrm{s}$ and the plasma frame moves equatorward with about $100 \mathrm{~m} / \mathrm{s}$. The existence of a proper motion of an arc in the plasma frame has been first observed by Haerendel et al. [1993] and later confirmed by Gazey et al. [1996] and Frey et al. [1996]. It was subsequently found that during the growth and recovery phases of a substorm the arcs drift with the plasma frame, but proper motions, sometimes quite strong or even in the opposite direction, exist during the expansion phase [Williams et al., 1998; del Pozo et al., 2002]. This proper motion is an essential ingredient of the "fracture model" of Haerendel [2007]. According to this model active arcs derive their energy from magnetic stress releases and thus propagate into the region of higher shear stresses, i.e., into the interior of the large-scale current system. The current sheets are therefore not moving with the plasma but become displaced like erosion fronts. The speed, $\mathrm{v}_{\mathrm{n}}$, of the proper motion is given by the width of current sheet or arc, divided by the energy conversion or erosion time, $\tau_{\text {arc }}$, which Haerendel [1994, 2007] estimated to be $4 \tau_{A}$ :

$$
\mathrm{v}_{n}=\frac{\mathrm{w}_{\text {arc }}}{\tau_{\text {arc }}} .
$$

There is a certain kinship between the powering of auroral arcs by propagating releases of shear stresses with the concept of magnetic energy advection by Knudsen et al. [2011]. However, in the latter treatment the proper motion of the current sheet with respect to the plasma or "cross-arc convection" remains undetermined.
[31] In the subsequent data evaluation we will determine $\tau_{\text {arc }}$ independently and obtain an experimental answer to a very central question of arc formation, namely how many reflections of Alfvén waves between arc and generator are needed to establish dynamical equilibrium. The here treated auroral event thus offers the unique possibility to check whether the theoretical proper motion according to equation (16) agrees with the conclusions from the optical and radar data.

[32] Turning now to the tangential electric field, we are quite aware that neither the motion of the plasma nor that of the arcs remains constant during the considered time interval. The poleward excursion of the Alfvénic arc between 04:01:30 UT and 04:02:30 UT may indicate a corresponding change of the motion of the plasma frame. However, we cannot exclude flux transfer from the polar cap through the Alfvénic arc. Furthermore, the observed variations of the arcs' brightness and motions may well be associated with changes of the electric field. Unfortunately, the Doppler data from the SuperDARN radar (Figure 7) are rather coarse. They indicate a motion of the plasma frame between 0 and $200 \mathrm{~m} / \mathrm{s}$. The equatorward motion of the Alfvénic arc of $100 \mathrm{~m} / \mathrm{s}$ for a period of $7 \mathrm{~min}$ appears to be consistent with the arc being frozen in the plasma frame. However, the average of the arc motion for the $10 \mathrm{~min}$ interval covered in Figure 4 was only $33 \mathrm{~m} / \mathrm{s}$. This raises doubts about the attachment of this arc to the plasma frame. (Zig-zag motions of the polar cap boundary are a common phenomenon during substorms possibly related to variations in the reconnection rate [Aikio et al., 2008]). Nevertheless, lacking more precise information it should be adequate for the aims of this investigation to idealize the situation by adopting an equatorward speed of the plasma of $100 \mathrm{~m} / \mathrm{s}$ or a westward directed tangential electric field of $5 \mathrm{mV} / \mathrm{m}$ measured in the frame of the conductivity enhancements in the $\mathrm{E}$ region, which is linked to the neutral atmosphere. Tacitly we assume that there is no motion of the neutral constituents with respect to the observer. The westward electric field leads to a poleward Hall current and an equatorward directed electric polarization field across the arcs driving a secondary Pedersen current which opposes the primary and dominant closure current (cf. Figure 11). Thus $E_{t}$ has to have a negative sign in equations (8) and (13). With the adopted value for $E_{t}$ we calculate $K, \mathrm{w}_{\mathrm{arc}}, \delta, \Sigma_{\mathrm{P}}, \Sigma_{\mathrm{H}}, \hat{\alpha}, R_{w}, L, \tau_{\text {arc }}$ and $\mathrm{v}_{\mathrm{n}}$. It needs only 3 iterations of $\hat{\alpha}$ and $R_{w}$ for convergence of the solution of equation (13). After $R_{w}$ has been obtained, one can derive $\tau_{\text {arc }}$ from equation (4) with the respective mean value of $L$ for each arc.

[33] One can immediately make some interesting observations. The Knight conductance, $K$, shows little variation with a tendency to increase equatorward, which is likely to be due to an increasing density of the generator plasma. The arc widths, except for that of arc 2, agree fairly well with the determinations from the satellite data. This is not at all trivial regarding the origin of equation (7). The excess of Hall versus secondary Pedersen current, $\hat{\alpha}$, turns out to range between 14 and $33 \%$. However, this amounts to rarely more than a $3 \%$ correction for the determination of $R_{w}$. There is no clear trend in the variation of $R_{w}$ or $\tau_{\text {arc }}$ with latitude. This is not really surprising since equations (13) and (4) contain a mix of inaccurately measured quantities and theoretical values. For the subsequent comparison with other environmental 
Table 2. Calculated Properties of Arcs and Environment ${ }^{\mathrm{a}}$

\begin{tabular}{|c|c|c|c|c|c|c|c|c|c|c|}
\hline Arc & $K\left(\mathrm{mho} / \mathrm{m}^{2}\right)$ & $\mathrm{w}_{\text {arc }}(\mathrm{km})$ & $\delta$ & $\Sigma_{\mathrm{P}}(\mathrm{mho})$ & $\Sigma_{\mathrm{H}}(\mathrm{mho})$ & $\hat{\alpha}$ & $R_{w}(\mathrm{ohm})$ & $L$ & $\tau_{\text {arc }}(\mathrm{s})$ & $\overline{v_{n}(m / s)}$ \\
\hline 1 & $1.02 \times 10^{-9}$ & 73.0 & 2.000 & 4.43 & 2.66 & 1.485 & 0.465 & 9.71 & 267.2 & 273.0 \\
\hline 2 & $0.83 \times 10^{-9}$ & 41.6 & 0.317 & 5.43 & 3.79 & 1.257 & 0.716 & 8.80 & 157.3 & 264.4 \\
\hline 3 & $1.17 \times 10^{-9}$ & 40.6 & 0.478 & 12.49 & 12.44 & 1.219 & 0.365 & 8.43 & 295.7 & 137.3 \\
\hline 4 & $1.45 \times 10^{-9}$ & 47.1 & 0.323 & 7.89 & 5.77 & 1.280 & 0.452 & 7.76 & 220.0 & 214.1 \\
\hline 5 & $1.53 \times 10^{-9}$ & 38.5 & 0.397 & 22.91 & 31.47 & 1.180 & 0.242 & 7.40 & 391.7 & 98.3 \\
\hline 6 & $1.59 \times 10^{-9}$ & 37.1 & 0.167 & 11.63 & 10.19 & 1.160 & 0.538 & 7.06 & 168.3 & 220.4 \\
\hline
\end{tabular}

${ }^{\mathrm{a} K n i g h t}$ conductance, $K$; arc width, $\mathrm{w}_{\text {arc }}$; ratio of field-aligned current to Pedersen current, $\delta$; Pedersen and Hall conductivities, $\Sigma_{\mathrm{P}}$ and $\Sigma_{\mathrm{H}}$; $\hat{\alpha}$ from equation (9); wave impedance, $R_{w}$, from equation (13); magnetic shell parameter, $L$, from equation (15); energy conversion time, $\tau_{\text {arc }}$, from equation (4); and speed of proper motion, $\mathrm{v}_{\mathrm{n}}$, from equation (16).

data we use the mean values of $\tau_{\text {arc }}$ and $\mathrm{v}_{\mathrm{n}}$, namely $250 \mathrm{~s}$ and $201 \mathrm{~m} / \mathrm{s}$. The "statistical" error of $\mathrm{v}_{\mathrm{n}}$ with six cases only amounts to $\pm 26 \mathrm{~m} / \mathrm{s}$.

[34] The evaluations listed in Table 2 rest on the adoption of a plasma frame speed of $100 \mathrm{~m} / \mathrm{s}$ southward or a westward electric field of $-5.0 \mathrm{mV} / \mathrm{m}$. This number is rather uncertain. The SuperDARN data are equally well consistent with $150 \mathrm{~m} / \mathrm{s}$ or $E_{t}=-7.5 \mathrm{mV} / \mathrm{m}$. For this reason we have recalculated $\hat{\alpha}, R_{w}, \tau_{\text {arc }}$, and $\mathrm{v}_{\mathrm{n}}$ in Table 3 with the latter value for $E_{t}$.

[35] The numbers listed in Table 3 exhibit the effect of the higher negative value for $E_{t}$, but the differences with those for $E_{t}=-5 \mathrm{mV} / \mathrm{m}$ in Table 2 are not great. The average energy conversion time is $258.5 \mathrm{~s}$ and the average proper motion $193.8 \mathrm{~m} / \mathrm{s} \pm 28 \mathrm{~m} / \mathrm{s}$. This shows that the impact of the plasma frame velocity on the derived proper motions is minimal. However, there is a significant difference in the total speed, plasma frame plus proper motion, the very speed that is optically observable. They are $(301 \pm 26) \mathrm{m} / \mathrm{s}$ and $(343.8 \pm 28) \mathrm{m} / \mathrm{s}$, respectively, while the observed mean speed of the arcs (excluding the Alfvénic arc) is (296 \pm $26 \mathrm{~m} / \mathrm{s}$ ). This yields a slight preference for our first choice of the speed of the plasma frame.

\subsection{Ionospheric Electrodynamic Properties}

[36] We can now continue exploiting the derived environmental properties and determine the currents flowing along the conductivity enhancements created by the arcs. This requires knowledge of the tangential electric field for which we adopted above $-5.0 \mathrm{mV} / \mathrm{m}$ or $-7.5 \mathrm{mV} / \mathrm{m}$. Greater precision is not possible.

[37] The Hall current driven by $E_{t}$ is not fully balanced by a Pedersen current. We have therefore:

$$
E_{x}=\frac{\Sigma_{H}}{\hat{\alpha} \Sigma_{P}} E_{t}+\frac{J_{P}}{\Sigma_{P}} .
$$

The westward $E_{t}$ appears here with a negative sign. Although the polarization field is opposed to the field,

Table 3. Same as Table 2 With $E_{t}=-7.5 \mathrm{mV} / \mathrm{m}$

\begin{tabular}{ccccc}
\hline Arc & $\hat{\alpha}$ & $R_{w}(\mathrm{ohm})$ & $\tau_{\text {arc }}(\mathrm{s})$ & $\mathrm{v}_{\mathrm{n}}(\mathrm{m} / \mathrm{s})$ \\
\hline 1 & 1.505 & 0.447 & 278.4 & 262.2 \\
2 & 1.266 & 0.690 & 163.4 & 254.6 \\
3 & 1.228 & 0.353 & 306.2 & 132.6 \\
4 & 1.291 & 0.436 & 228.2 & 206.4 \\
5 & 1.183 & 0.238 & 398.7 & 96.6 \\
6 & 1.167 & 0.513 & 176.2 & 210.6 \\
\hline
\end{tabular}

$E_{x, o}=J_{P} / \Sigma_{P}$, driving the primary Pedersen current (see Figure 11), it turns out that the latter component is dominant and $E_{x}$ is only weakened inside the arcs without changing sign. We have the situation designated as "anticorrelation arc" by de la Beaujardière et al. [1981] and specifically as "evening anticorrelation arc" by Marklund [1984]. The height integrated current density along the arcs consists of an eastward Hall current driven by the poleward pointing residual $E_{x}$ and a westward Pedersen current driven by $E_{t}$, whereby the first component dominates:

$$
J_{y}=s J_{P}+\left(1+\frac{s^{2}}{\hat{\alpha}}\right) \Sigma_{P} E_{t}
$$

The total (eastward) current along an arc thus becomes:

$$
I_{y}=J_{y} \mathbf{w}_{\text {arc }} .
$$

Finally, we are interested in the ionospheric dissipation:

$$
\dot{W}_{\text {ion }}=\Sigma_{P}\left(E_{x}^{2}+E_{t}^{2}\right) .
$$

The respective values for each arc have been assembled in Table 4 as well as the ratio of the energy conversion rate by the auroral process over the total converted energy flux.

[38] The reduction of $E_{x}$ by the electric polarization field is clearly visible in arcs 3 and 5 which have higher energy fluxes and create a larger secondary ionization. The current strengths reflect the different brightnesses and widths of the arcs. There is a clear trend in the latitudinal variation of the total energy conversion rate. It reflects the increase of the Pedersen current toward the center of the large-scale Type II current system and the related increase of free energy by the sheared field. The strongly different auroral

Table 4. Ionospheric Electrodynamic Parameters of the Arcs, Ionospheric and Total Dissipation Rates, Ratio of Auroral and Total Energy Conversion Rates

\begin{tabular}{ccccccc}
\hline $\begin{array}{c}E_{x} \\
\text { Arc }\end{array}$ & $\begin{array}{c}J_{y} \\
(\mathrm{mV} / \mathrm{m})\end{array}$ & $\begin{array}{c}I_{y} \\
{[\mathrm{kA}]}\end{array}$ & $\begin{array}{c}\dot{W}_{\text {ion }} \\
\left(\mathrm{mW} / \mathrm{m}^{2}\right)\end{array}$ & $\begin{array}{c}\dot{W}_{\text {tot }} \\
\left(\mathrm{mW} / \mathrm{m}^{2}\right)\end{array}$ & $\dot{W}_{\text {arc }} / \dot{W}_{\text {tot }}$ \\
\hline 1 & 9.8 & 0.004 & 0.28 & 0.53 & 2.55 & 0.790 \\
2 & 27.2 & 0.076 & 3.15 & 4.14 & 6.01 & 0.386 \\
3 & 16.2 & 0.139 & 5.63 & 3.58 & 11.17 & 0.680 \\
4 & 44.6 & 0.218 & 10.26 & 15.89 & 20.43 & 0.222 \\
5 & 18.3 & 0.462 & 17.77 & 8.21 & 29.47 & 0.716 \\
6 & 62.6 & 0.580 & 21.50 & 45.87 & 53.51 & 0.142 \\
\hline
\end{tabular}


energy conversion rates combined with this trend lead to strong variations of the ratio of the two processes.

[39] We refrain from listing the numbers for the electrodynamic parameters corresponding to the choice of $E_{t}=$ $-7.5 \mathrm{mV} / \mathrm{m}$, since the deviations from those of Table 4 are not significant.

\section{Data Interpretation}

\subsection{Proper Motions}

[40] The most important conclusion to be derived from Table 2 is that the calculated proper motions of the arcs turn out to be consistent with the conclusions from the differences between the measured equatorward drifts of plasma and arcs. As was to be expected, there is quite some variation in the proper motions since they are governed by arc width and Alfvénic transit time, the first depending on the efficiency of the energy dumping, the second on the magnetospheric density distribution. However, this consistency is most significant, since the derived magnitude of $v_{n}$ is essentially owed to the theoretical concept that the energy extracted from the sheared magnetic field is consumed by auroral acceleration and ionospheric dissipation during the time that the arc needs to move by its width. Therefore it is most satisfying that the resulting average proper motion of $200 \mathrm{~m} / \mathrm{s}$ agrees astonishingly well with the observed arc motions in the reference frame of the plasma, whereby the precise magnitude of the latter turns out to be not critical. Beyond supporting the general concept, this result also allows a determination of the arc-generator interaction time scale and confrontation with the four Alfvénic transit times proposed by Haerendel [1994, 2007]. It is therefore worthwhile to realize how and where this time scale enters into the derived quantities.

[41] The definition of the proper motion in equation (16) involves the time scale of $\tau_{\text {arc }}$ and the derived arc width. The latter follows from equation (7) and contains only observed electrodynamic and energetic quantities. $\tau_{\text {arc }}$ is derived from $R_{w}$ through equation (4). Equation (13), from which $R_{w}$ is derived, also contains only observables or quantities like $K$ and $\hat{\alpha}$ which are also derived from observed quantities. So, the time scale does not enter anywhere arbitrarily but is intrinsic to the theoretical concept. This is perhaps best seen when one expresses $\mathrm{v}_{\mathrm{n}}$ directly by $R_{w}$ using equations (4) and (16):

$$
\mathrm{v}_{n}=\frac{\mathrm{w}_{\text {arc }} R_{w}}{\mu_{0} \ell_{e f f}} .
$$

The expression for the effective length of the field line contains two quantities, $L$ and $\Gamma^{2}$ that are computed on the basis of the distorted dipole model with a high-beta generator plasma. Looking at Table 2, the values for $L$ calculated with equation (15) appear to be very realistic. For instance, the ionospheric foot point of the THEMIS-A location at a distance of $7.6 \mathrm{R}_{\mathrm{E}}$, as mapped by using the Tsyganenko [1989] model, is found at a latitude close to that of arcs 4 and 5, as one can check by comparing Figures 2 and 8 . For $\Gamma^{2}$ the value of 1.25 applying to $\beta_{e q}=2$ has been adopted in evaluating equation (4). In conclusion we see that the magnitude of the derived proper motion emerges directly from data and model parameters and thus determines the interaction time scale:

$$
\tau_{\text {arc }}=\frac{w_{\text {arc }}}{\mathrm{v}_{n}} .
$$

The average of $\tau_{\text {arc }}$ calculated with the numbers of Table 2 yields $250 \mathrm{~s}$. This number offers the possibility to check whether the proposition of Haerendel [1994, 2007] that $\tau_{\text {arc }} \approx 4 \tau_{A}$ is supported by these data. This needs an independent assessment of $\tau_{A}$. We will address this question in the next subsection.

\subsection{Interaction Time Scale}

[42] As satisfying as the agreement of derived and observed proper motions is, one must not overlook the large scatter of the values for $R_{w}$ and thus for $\tau_{\text {arc }}$ for the individual arcs (Figure 12). According to equation (4) $R_{w}$ should vary monotonically if $\tau_{\text {arc }}$ would simply reflect the travel time of an Alfvén wave between ionosphere and generator. The differential magnetic energy density, 6th column in Table 1, varies rather smoothly. Since $R_{w}$ is derived from the energy conservation equations (12) and (13), one should expect little fluctuation. Also the arc widths which enter that relation show no great variations. However, the auroral energy flux, $\dot{W}_{\text {arc }}$, is far from varying smoothly with latitude, and so is the related ionospheric dissipation rate. Furthermore, one observes strong brightness and structural variations of the arcs, such as brightness fading and arc splitting and unfolding. We must therefore conclude that the energy flux out of the magnetosphere is subject to temporal and local variations of parameters not appearing in the quasistationary theoretical model underlying the applied formalism and manifesting themselves through variations of $\tau_{\text {arc }}$. The FAST data, representing a snapshot of the properties of the individual arc, may not be fully representative of its mean properties for which the theoretical framework has been derived, because the contact time is much shorter than the interaction time between arc and generator. For instance, the low value of $R_{w}$ and high value of $\tau_{\text {arc }}$ for arc 5 are clearly related to the high numbers for the ionospheric conductivities, which originate from the high auroral energy flux. Looking at Figure 2 one can verify that at the time of the FAST crossing the arc was undergoing substantial brightening, broadening and subsequent arc splitting. Such dynamic behavior is certainly not foreseen in the quasistationary theory. In other words, the large scatter of $\tau_{\text {arc }}$ reflects the real situation.

[43] It would be ideal for the interpretation of $\tau_{\text {arc }}$ if we had an independent measure of the travel time of an Alfvén wave between ionosphere and equator. Geomagnetic pulsations in the Pc 4 range would be the obvious candidates, in particular those polarized in the east-west direction, the same way as the shear component of the Type II current system when it becomes decoupled by the auroral process. However, Pc 4 pulsations have been found to be poloidal and partially compressional as well as azimuthal modes [Kokubun et al., 1989]. The toroidal Pc 4 waves are the fundamental mode with a magnetic node and an electrical field antinode at the equator. The poloidal waves, on the other hand, must be even modes, i.e., second harmonic. Samson et al. [1971] have 
determined the latitudinal dependence of quasi-monochromatic micro pulsations in the general area of this investigation at nighttime and found concentrations of $7-15 \mathrm{mHz}$ pulsations at the latitude of Ft. Smith, which is similar to that of Ft. Simpson, with strong polarization in the D-direction. Nosé et al. [1998] were able to clearly establish the existence of azimuthal Pc 4 pulsations during nighttime by measurements with the Engineering Test Satellite (ETS-VI). They found the occurrence correlated with substorm onset. The dashed line in Figure 12 (taken from Nosé et al. [1998, Figure 7]) represents twice the eigenperiods of standing azimuthal Alfvén waves as calculated with the wave equation of Cummings et al. [1969]. AMPTE CCE measurements of the anisotropy of ion fluxes and of the magnetic field by Takahashi et al. [2002] at L $=6-10$ and slightly off the equatorial plane revealed the existence of toroidal standing Alfvén waves at all local times. They found median frequencies from 6 to $10 \mathrm{mHz}$ at $\mathrm{L}=7$ and from 4 to $8 \mathrm{mHz}$ at $\mathrm{L}=9$, the range of $\mathrm{L}$-values of our arc event. Figure 12 shows that Takahashi et al. [2002] used these wave frequencies for determining equatorial plasma densities by fitting with empirical density and magnetic field models and obtained for the early night hours densities between 1.5 and $3.0 \mathrm{~cm}^{-3}$, rather close to the THEMIS-A data for our event. Taking the mean of the measured frequency range, i.e. $f_{m}=6.5 \mathrm{mHz}$, we find an Alfvénic travel time from ionosphere/ acceleration region to the equator of $\tau_{A}=1 /\left(4 f_{m}\right)=38.5 \mathrm{~s}$. Takahashi et al. [2002] calculated model variations of the fundamental azimuthal frequencies on the basis of these data. Figure 12 shows the results for 19:00 MLT and $\mathrm{Kp}=2$, whereby L was converted to MLAT by means of equation (15).

[44] We also can calculate the Alfvénic transit time from ionosphere to the equator, $\tau_{A}$, directly using density and magnetic field readings at THEMIS-A under the assumption of an isotropic density distribution at the equator and thus little variation along $\mathrm{B}$ following the formalism of the distorted dipole model of [Haerendel, 2007]:

$$
\tau_{A}=\frac{\sqrt{\mu_{0} \rho}}{B_{s}} \cdot L^{4} \cdot \int_{0}^{\zeta_{i o n}}\left(1-\zeta^{2}\right)^{3} \cdot \sqrt{1+\beta_{e q} / b^{2}(\zeta)} d \zeta
$$

with $B_{s}=0.3 \mathrm{G}, \zeta=\sin \lambda$, and $b(\zeta)=B(\zeta) / B_{e q}$ along a field line. For the latitude of FSIM the integral amounts to 0.655 . Taking from THEMIS-A an equatorial hydrogen density of $1.3 \mathrm{~cm}^{-3}$ and L from equation (15) with $\lambda_{F S I M}=67.3^{\circ}$ yields $\tau_{A}=31 \mathrm{~s}$. In reality, the density may increase toward Earth and thus prolong the transit time. For instance, Goldstein et al. [2001] find a variation $\mathrm{n}_{\mathrm{e}} \sim \mathrm{r}^{-1.7}$ in the density trough. However, since the low magnetic field magnitude near the equator dominates the Alfvénic propagation speed, the correction for $\tau_{A}$ stays below $15 \%$. A further aspect is the well-known existence of plasma density cavities above auroral acceleration regions extending over a wide range of altitudes down to even $1000 \mathrm{~km}$ [Benson and Calvert, 1979; Persoon et al., 1988; Lundin et al., 1994], often dominated by hot magnetospheric electrons [Strangeway et al., 1998]. Such cavities suggest a low density gradient between equator and low magnetosphere and, as a consequence, a rather rapid density increase toward the middle and lower ionosphere, so that the reflection of an Alfvén wave can be essentially regarded as reflection from a discontinuity.
[45] In conclusion, measurements and direct calculations for the event conditions yield the rather narrow range of 30 to $39 \mathrm{~s}$ for $\tau_{A}$. This means that the mean interaction time of an arc with the current generator ranges between 7 to 8 Alfvénic transit times. This is a most significant result, since it is the first empirical determination of $\tau_{\text {arc }}$ and a correction of Haerendel's [1994, 2007] adoption of $4 \tau_{A}$, which was actually regarded as a lower limit. In numerical solutions of a 2D MHD model, Lysak and Dum [1983] found a need for five or more wave bounce periods before steady state was reached, albeit for a different situation. On the other hand, values as low as $5 \tau_{A}$ or as high as $12 \tau_{A}$ are suggested by the individual data. One may thus conclude that $\tau_{\text {arc }}$ has a minimum of about five Alfvénic transit times, but much longer energy conversion times are possible due to variations of the energy source.

\subsection{The Field-Aligned Conductance}

[46] THEMIS-A plasma data from plasma obtained not too far away from the source region of the observed arcs allow us to calculate the Knight constant:

$$
K=\frac{e^{2} n_{H}}{\sqrt{\pi} n_{H} c_{e H}} .
$$

The hot electron density, $n_{H}$, was found to be $1.3 \mathrm{~cm}^{-3}$ and the parallel electron temperature $2.5 \mathrm{keV}$ during the interval of the FAST crossing. This yields a thermal electron speed $c_{e H}=2.96 \mathrm{~cm} / \mathrm{s}$ and $\mathrm{K}=7.0 \times 10^{-10} \mathrm{mho} / \mathrm{m}^{2}$. Comparison with the average value of $\mathrm{K}$ shows that the actual conductance was about a factor of 2 higher. This should not be regarded as a discrepancy, not even as surprising, since even stronger disagreements have been found by Sakanoi et al. [1995] from measurements of the Akebono spacecraft. These authors derived electron density and temperature from fitting the locally measured energy spectra to an isotropic Maxwellian distribution. Part of the discrepancy may be due to the contribution of trapped electrons to the conductance derived from measurements, another one from an overestimate of the electron temperature. We can thus safely conclude that the calculated conductance is consistent with the values shown in Table 2 .

\subsection{Magnetic Perturbations by Currents Along the Arcs}

[47] Using the total current intensities in Table 4 and the geometric relations relative to FSIM one finds that the nearest arc, arc 3, must have contributed $4.4 \mathrm{nT}$ and the strongest arc, arc 5, $7.6 \mathrm{nT}$ to the horizontal perturbations at FSIM. These low values are due to the reduction of the Hall current inside the arcs by the westward electric field (see Figure 11), a situation opposite to the more familiar one of the westward electrojet which is enhanced by the Cowling effect and the polarization field across the arc. The at first sight surprising finding of an almost vanishing horizontal component at FSIM underneath quite prominent arcs is thus due to a negative Cowling effect. A similar reduction of electric field and current was observed by de la Beaujardière et al. [1981]. The measured magnitude of about $20 \mathrm{nT}$ at 04:02 UT is partially due to the main eastward electrojet located south of FSIM near the center of the Type II current system, as evident from the strong negative Z-component 
(see Figure 1, eighth panel) and also supported by the distribution of the equivalent currents shown in Figure 8. The magnitudes of the integrated currents, $\mathrm{J}_{\mathrm{y}}$, along the arcs as listed in Table 4 agree also well with those of the equivalent currents shown in Figure 8.

\section{Birth and Life of the Arcs}

[48] It was most fortunate that the short interval of the FAST crossing over the FOV of the all sky camera at FSIM was imbedded in a much longer period of auroral observations. During this time we could track the equatorward drift of the arcs as well as several first appearances of arcs adjacent to the Alfvénic arc at the polar border of the oval. During the half hour from 03:45 UT to 04:15 UT we could observe the appearance of seven arcs. They appeared with very low brightness and moved quickly equatorward with a speed similar to that documented for the 'new arc' in Figure 4. The arcs gained substantial intensity once they reached zenith over the FSIM all sky imager. This is well documented by the all sky images of Figure 2 and reflected by the monotonic increase of the total available energy, $\left(B_{\perp u}^{2}-B_{\perp d}^{2}\right) / 2 \mu_{0}$, with decreasing latitude in Table 1. Our interpretation is that the arcs do not deplete the free energy stored in the sheared field and it appears that it is constantly replenished, however not necessarily at a constant rate.

[49] The general increase of brightness as the arcs proceed equatorward is by no means monotonic. This can be observed in the examples of all sky images in Figure 2 and the keograms of Figure 3. Obviously the energy pumping into the gross current system is subject to variations, temporally and spatially. This is not at all surprising, since the event occurred in a long period of high auroral activity (see first panel of Figure 1) with substorms proceeding further to the east. Approximately $10 \mathrm{~min}$ after the interval under investigation a westward traveling surge arrived in the FOV of the all sky camera at Fort Smith about $500 \mathrm{~km}$ east of FSIM. Substorms are known to evolve with stepwise injections or activations typically every five minutes [Sergeev et al., 2000]. Such activations are probably behind the variable pumping of energy into the oval during the early evening hours of our event. The reversal of the slow equatorward motion of the Alfvénic arc between 04:01:30 and 04:02:30 UT shown in Figure 4 was most likely the result of an increased energy input into the evening oval.

[50] Another manifestation of variable energy input is the occasional brightening, broadening, splitting, and unfolding of an arc. This happened for instance during the interval for 03:58:00 to 03:58:48 UT (Figure 5). The splitting proceeded on the equatorward side of the arc and the westward progression of the created fold had a speed of about $3 \mathrm{~km} / \mathrm{s}$. The values of $E_{x}$ listed in Table 3 are mostly well below $50 \mathrm{mV} / \mathrm{m}$ corresponding to $1.0 \mathrm{~km} / \mathrm{s}$ westward velocity. This shows that the unfolding motion is not shared by the ionospheric plasma but clearly a matter of the plasma and field dynamics at and above the acceleration region. In this context it is interesting to note that similar splitting and unfolding could be observed at the Alfvénic arc, but the splitting occurred at the poleward side and the unfolding proceeded eastward. This deviation from the arcs' behavior may be intimately related to the generally eastward motion of the rays of that arc in contrast to the westward motions prevailing equatorward of it.

[51] The observation of steady production of new arcs at the poleward boundary of the auroral oval and the subsequent equatorward motion under brightening raises the question where this process comes to an end. Where do auroral arcs disappear? It is obvious, at least in the framework of arcs feeding on the release of shear stresses, that the equatorward motion must die out at the transition from upward to downward field-aligned currents. For a pure Type II system, this coincides with the maximum of the Pedersen current. Indeed, the FAST data in Figure 10 show an arc, namely arc 6, which is in direct contact with a broad region of downward current with all the signatures of the upward accelerated, low-energy electrons and transversely heated ions. Arc 6 is probably disappearing, unfortunately invisible to the FSIM all sky camera. It could not proceed any further equatorward, because unstressing the sheared field at its leading edge would reduce the field-aligned current rather than enhancing it as in the poleward region of equatorward increasing shear fields. We will return to this matter in the conclusions section. It will remain a mystery what the nature of the electron event after 04:04:20 UT is due to.

\section{Conclusions}

[52] The event of the 09 March 2008 pass of FAST over the all sky FOV at Ft. Simpson/Canada offers one of the rare opportunities in auroral physics for a critical examination of the predictions of a theoretical model. The reason for that is threefold: (1) the relatively clear realization of a Type II current system, (2) the presence of several arcs with traceable motions, and (3) an almost complete data set from the spacecraft (only electric field data on FAST are missing). The first point contains the fact that there is a broad region of upward field-aligned current (about 6 degrees in latitude) not interrupted by sheets of downward current. Second, embedded in it are sheets of intensified field-aligned currents subject to the auroral energy conversion process. Hence the arcs are part of a wider current system, not separate entities like narrow propagating waves. Third, almost all of the most important auroral parameters are covered by FAST data and ground observations. A critical input for checking the theory is the combined optical and radar observations. They present evidence for the existence of proper motions of the arcs in the plasma frame, which is the heart of the theoretical concept. Proper motions are indicators of the progressing release of shear stresses which constitutes the energy source and can be related to the auroral energy flux plus the ionospheric energy dissipation rate. As discussed in the context of equation (21), the theoretical proper motion follows practically only from parameters deduced from observables such as the energy flux and currents with only two noncritical model parameters and the empirical formulas for the calculation of the ionospheric conductivities. The verification of the existence of proper motions and the close quantitative agreement of the average derived speed with the observed one is the most important result of this study. The quantitative connection of proper motion and energy conversion in arc formation and ionosphere is clear evidence that the motion is not impressed by the generator. 
[53] The second important finding is a determination of the time, $\tau_{\text {arc }}$, needed for establishing some sort of equilibrium between the energy source and the arc. It turns out to take on average about four minutes. Depending on the somewhat uncertain length of the communication period, $2 \tau_{A}$, between generator plasma and auroral acceleration region, it ranges between 6 and $8 \tau_{A}$. This time is needed to extract the available free energy and determine the optimum arc width $\left(\mathrm{w}_{\text {arc }}=\tau_{\text {arc }} \cdot \mathrm{v}_{n}\right)$. However, large deviations from the average exist which are attributed to temporal variations of the energy source. In view of these deviations it is not at all trivial that the arc widths determined from sheet current density, energy flux and mean electron energy agree well with the widths determined from the satellite crossing times.

[54] Even more remarkable is that a quasi-stationary theoretical framework appears to reproduce the basic behavior of auroral arcs with intrinsic time scales that are much longer than the actually observed fluctuations. For instance, the arc broadening, splitting, and unfolding with fast westward speed observed after 03:57:48 UT occurred within about one minute. Also brightness changes are observed within much shorter time spans than the total interaction time scale, $\tau_{\text {arc }}$, which is of the order of four minutes. Also the reversal of the slow equatorward motion of the poleward border of the oval marked by the Alfvénic arc lasted for only one minute. Some of these changes have a quasiperiodic character, as one can recognize when following the equatorward drifting traces in the keogram (Figure 3). However, these periods range around $160 \mathrm{~s}$, i.e., more than twice as long as one communication period, $2 \tau_{A}$, between arc and source plasma. A simple model with bouncing Alfvén waves would not match these data. The observed variations must essentially reflect the dynamics of the source plasma and a variable energy input into the large-scale current and convection system.

[55] The conclusion from all this must be that the basic theoretical concept, extraction of energy from the sheared field to feed the arc, is preserved within a real situation with strongly modulated energy source and thereby modulated interaction of generator plasma and auroral arcs. In the underlying quasi-stationary model, the width of the arc follows from the assumption of perfect matching between electromagnetic energy inflow with the energy conversion by the auroral acceleration process and ionospheric dissipation. The persistence of individual arcs over many communication periods, $2 \tau_{\mathrm{A}}$, and brightness variations seem to imply that the principle of perfect matching and thus optimum energy conversion [see Haerendel, 2012] is rather quickly established in M-I coupling.

[56] A side result of this study is the quantitative verification of the surprising finding that the observed arcs created almost negligible magnetic perturbations at the station underneath. This is to be attributed to the fact that the westward electric field reduces the Hall current along the arcs instead of enhancing it, when the dominate convection field is poleward.

[57] An unavoidable consequence of the concept, supported by this study, that arcs derive their energy from the progressive release of magnetic shear stresses is the finite life time of individual arcs. Born at the outer edge of the upward current region and proceeding deeper and deeper into the center of the stored magnetic energy, they must die out when further progression would lead to negative energy supply. Taking the average proper motion and the latitudinal extent of the upward current region of about $660 \mathrm{~km}$, we derive an average life time of about one hour for the situation during the observed event. This takes into account the continuous supply of energy as expressed in the long average value of $\tau_{\text {arc }}$ and the continued formation of new arcs at the polar border of the oval. Unfortunately, the dying arc was outside the FOV of the FSIM all sky camera thus not allowing us to observe the expected brightness decay. However, there should be plenty of opportunity to study this process in view of the availability of long periods of auroral observations with high temporal resolution by the THEMIS GBO network.

[58] Acknowledgments. The work on this topic started during an ISSI meeting on small-scale auroral structures and the authors are grateful to the International Space Sciences Institute for providing the opportunity and the infrastructure. We acknowledge NASA contract NAS5-02099 and V. Angelopoulos for use of data from the THEMIS Mission, S. Mende and E. Donovan for use of the ASI data, the CSA for logistical support in fielding and data retrieval from the GBO stations, and NSF for support through award 1004736 for GIMNAST. The work by Chris Chaston was supported by NSF grant AGS-1102514. GH thanks Forrest S. Mozer and Robert P. Lin for their support of this work.

[59] Robert Lysak thanks the reviewers for their assistance in evaluating this paper.

\section{References}

Aikio, A. T., T. Pitkänen, D. Fontaine, I. Dandouras, O. Amm, A. Kozlovsky, A. Vaivads, and A. Fazakerley (2008), EISCAT and Cluster observations in the vicinity of the dynamical polar cap boundary, Ann. Geophys., 26, 87-105, doi:10.5194/angeo-26-87-2008.

Amm, O. (1997), Ionospheric elementary current systems in spherical coordinates and their application, J. Geomagn. Geoelectr., 49, 947-955, doi:10.5636/jgg.49.947.

Amm, O., and A. Viljanen (1999), Ionospheric disturbance magnetic field continuation from the ground to the ionosphere using spherical elementary current systems, Earth Planets Space, 51, 431-440.

Amm, O., M. J. Engebretson, T. Hughes, L. Newitt, A. Viljanen, and J. Watermann (2002), A travelling convection vortex event study: Instantaneous equivalent currents, estimation of field-aligned currents, and the role of induced currents, J. Geophys. Res., 107(A11), 1334, doi: $10.1029 / 2002 J A 009472$

Angelopoulos, V. (2008), The THEMIS Mission, Space Sci. Rev., 141, 5-34, doi:10.1007/s11214-008-9336-1.

Benson, R. F., and W. Calvert (1979), ISIS 1 observations of the source of auroral kilometric radiation, J. Geophys. Res., 6, 479-482.

Boström, R. (1964), A model of the auroral electrojets, J. Geophys. Res., 69, 4983-4999.

Carlson, C. W., R. F. Pfaff, and J. G. Watzin (1998), The Fast Auroral SnapshoT (FAST) mission, Geophys. Res. Lett., 25, 2013-2016, doi:10.1029/98GL01592.

Cummings, W. D., R. J. O’Sullivan, and P. J. Coleman Jr. (1969), Standing Alfvén waves in the magnetosphere, J. Geophys. Res., 74, 778-793, doi:10.1029/JA074i003p00778.

de la Beaujardière, O., R. Vondrak, R. Heelis, W. Hanson, and R. Hoffman (1981), Auroral arc electrodynamic parameters by AE-C and the Chatanika radar, J. Geophys. Res., 86, 4671-4685, doi:10.1029/ JA086iA06p04671.

de la Beaujardière, O., L. R. Lyons, J. M. Ruohoniemi, E. Friis-Christensen, C. Danielsen, F. R. Rich, and P. T. Newell (1994), Quiet-time intensifications along the poleward auroral boundary near midnight, J. Geophys. Res., 99, 287-298, doi:10.1029/93JA01947.

del Pozo, C. F., P. J. S. Williams, N. J. Gazey, P. N. Smith, F. Honary, and M. Kosch (2002), Multi-instrument observations of the dynamics of auroral arcs: A case study, J. Atmos. Sol. Terr. Phys., 64, 1601-1616, doi:10.1016/S1364-6826(02)00083-4.

Echim, M. M., R. Maggiolo, M. Roth, and J. De Keyser (2009), A magnetospheric generator driving ion and electron acceleration and electric currents in a discrete auroral arc observed by Cluster and DMSP, Geophys. Res. Lett., 36, L12111, doi:10.1029/2009GL038343.

Elphinstone, R. D., J. S. Murphree, and L. L. Cogger (1996), What is a global auroral substorm?, Rev. Geophys., 34, 169-232, doi:10.1029/ 96RG00483. 
Frey, H. U., et al. (1996), Optical and radar observations of the motion of auroral arcs, J. Atmos. Terr. Phys., 58, 57-69, doi:10.1016/00219169(95)00019-4

Frey, H. U., et al. (2010), Small and meso-scale properties of a substorm onset auroral arc, J. Geophys. Res., 115, A10209, doi:10.1029/ 2010JA015537.

Fujii, R., R. A. Hoffman, P. C. Anderson, J. D. Craven, M. Sugiura, L. A. Frank, and N. C. Maynard (1994), Electrodynamic parameters in the nighttime sector during auroral substorms, J. Geophys. Res., 99, 6093-6112, doi:10.1029/93JA02210.

Fujii, R., O. Amm, A. Yoshikawa, A. Ieda, and H. Vanhamäki (2011), Reformulation and energy flow of the Cowling channel, J. Geophys. Res., 116, A02305, doi:10.1029/2010JA015989.

Fukushima, N. (1976), Generalized theorem for no ground magnetic effect of vertical currents connected with Pedersen currents in the uniformconductivity ionosphere, Rep. Ionos. Space Res. Jpn., 30, 35-40.

Gazey, N. G. J., P. N. Smith, R. P. Rijnbeek, M. Buchan, and M. Lockwood (1996), The motion of auroral arcs within convective plasma flow, Eur Space Agency Spec. Publ., ESA SP-389, 11-16.

Goldstein, J., R. E. Denton, M. K. Hudson, E. G. Miftakhova, S. L. Young, J. D. Menietti, and D. L. Gallagher (2001), Latitudinal density dependence of magnetic field lines inferred from Polar plasma data, J. Geophys. Res., 106(A4), 6195-6201, doi:10.1029/2000JA000068.

Haerendel, G. (1980), Auroral particle acceleration-An example of a universal plasma process, ESA J., 4, 197-204.

Haerendel, G. (1994), Acceleration from field-aligned potential drops, Astrophys. J. Suppl. Ser., 90, 765-774, doi:10.1086/191901.

Haerendel, G. (2007), Auroral arcs as sites of magnetic stress release, J. Geophys. Res., 112, A09214, doi:10.1029/2007JA012378.

Haerendel, G. (2009), Poleward arcs of the auroral oval during substorms and the inner edge of the plasma sheet, J. Geophys. Res., 107, A06214, doi:10.1029/2009JA014138.

Haerendel, G. (2012), A tool for characterizing and evaluating Type II auroral arcs, J. Geophys. Res., 117, A06214, doi:10.1029/2012JA017523.

Haerendel, G., S. Buchert, C. L. LaHoz, B. Raaf, and E. Rieger (1993), On the proper motion of auroral arcs, J. Geophys. Res., 98, 6087-6099, doi:10.1029/92JA02701.

Hallinan, T., and T. Davis (1970), Small-scale auroral arc distortions, Planet. Space Sci., 18, 1735, doi:10.1016/0032-0633(70)90007-3.

Hamrin, M., et al. (2006), Observations of concentrated generator regions in the nightside magnetosphere by Cluster/FAST conjunctions, Ann. Geophys., 24, 637-649, doi:10.5194/angeo-24-637-2006.

Hasegawa, H., N. Ohno, and T. Sato (2010), Simulation of feedback instability in the coupled magnetosphere-ionosphere system, J. Geophys. Res. 115, A08304, doi:10.1029/2009JA015093.

Kan, J. R., R. L. Williams, and S.-I. Akasofu (1984), A mechanism for the westward traveling surge during substorms, Baumjohann, J. Geophys. Res., 89, 2211-2216, doi:10.1029/JA089iA04p02211.

Karlsson, T., G. T. Marklund, S. Figueiredo, T. Johansson, and S. Buchert (2004), Separating spatial and temporal variations in auroral electric and magnetic fields by Cluster multipoint measurements, Ann. Geophys., 22, 2463-2472, doi:10.5194/angeo-22-2463-2004.

Keiling, A., J. R. Wygant, C. A. Cattell, F. S. Mozer, and C. T. Russell (2003), The global morphology of wave Poynting flux: Powering the aurora, Science, 299, 383, doi:10.1126/science.1080073.

Knight, S. (1973), Parallel electric fields, Planet. Space Sci., 21, 741-750, doi:10.1016/0032-0633(73)90093-7.

Knudsen, D. J., J. K. Burchill, E. F. Donovan, and V. M. Uritsky (2011), Advection of magnetic energy as a source of power for auroral arcs, Geophys. Res. Lett., 38, L24103, doi:10.1029/2011GL049661.

Kokubun, S., K. N. Erickson, T. A. Fritz, and R. L. McPherron (1989), Local time asymmetry of Pc 4-5 pulsations and associated particle modulations at synchronous orbit, J. Geophys. Res., 94, 6607-6625, doi:10.1029/JA094iA06p06607.

Lotko, W., A. V. Streltsov, and C. W. Carlson (1998), Discrete auroral arc, electrostatic shock and suprathermal electrons powered by dispersive, anomalously resistive field line resonance, Geophys. Res. Lett., 25, 4449-4452, doi:10.1029/1998GL900200.

Lundin, R., L. Eliasson, G. Haerendel, M. Boehm, and B. Holback (1994), Large-scale auroral plasma density cavities observed by Freja, Geophys. Res. Lett., 21, 1903-1906, doi:10.1029/94GL00888.

Lysak, R. L., and C. T. Dum (1983), Dynamics of magnetosphereionosphere coupling including turbulent transport, J. Geophys. Res., 88, 365, doi:10.1029/JA088iA01p00365.

Lysak, R. L., and W. Lotko (1996), On the kinetic dispersion relation for shear Alfvén waves, J. Geophys. Res., 101(A3), 5085-5094, doi:10.1029/ 95JA03712.

Lysak, R. L., and Y. Song (2002), Energetics of the ionospheric feedback instability, J. Geophys. Res., 107(A8), 1160, doi:10.1029/2001JA000308.
Marghitu, O., B. Klecker, G. Haerendel, and J. McFadden (2004), ALADYN: Method to investigate auroral arc electrodynamics from satellite data, J. Geophys. Res., 109, A11305, doi:10.1029/2004JA010474

Marghitu, O., M. Hamrin, B. Klecker, K. Rönnmark, S. Buchert, L. M. Kistler, M. André, and H. Rème (2006), Experimental investigation of auroral generator regions with conjugate Cluster and FAST data, Ann. Geophys., 24, 619-635, doi:10.5194/angeo-24-619-2006.

Marklund, G. (1984), Auroral arc classification scheme based on the observed arc-associated electric field patterns, Planet. Space Sci., 32, 193-211, doi:10.1016/0032-0633(84)90154-5.

Mende, S. B., C. W. Carlson, H. U. Frey, L. M. Peticolas, and N. Østgaard (2003), FAST and IMAGE-FUV observations of a substorm onset, J. Geophys. Res., 108(A9), 1344, doi:10.1029/2002JA009787.

Mende, S. B., et al. (2008), The THEMIS array of ground-based observatories for the study of auroral substorms, Space Sci. Rev., 141, 357-387, doi:10.1007/s11214-008-9380-x.

Nosé, M., T. Iyemori, S. Nakabe, H. Matsumoto, and T. Goka (1998), ULF pulsations observed by the ETS-VI satellite: Substorm associated azimuthal Pc 4 pulsations on the nightside, Earth Planets Space, 50, 63-80.

Oguti, T. (1974), Rotational deformations and related drift motions of auroral arcs, J. Geophys. Res., 79, 3861-3865, doi:10.1029/JA079i025p03861.

Oguti, T. (1975), Two-tiered auroral bands, J. Atmos. Terr. Phys., 37, 1501, doi:10.1016/0021-9169(75)90082-3.

Paschmann, G., S. Haaland, and R. Treumann (2002), Auroral Plasma Physics, Space Sci. Ser., vol. 103, 485 pp., Springer, New York.

Persoon, A. M., D. A. Gurnett, W. K. Peterson, J. H. Waite Jr., J. L. Burch, and J. L. Green (1988), Electron density depletions in the nightside auroral zone, J. Geophys. Res., 93, 1871-1895, doi:10.1029/JA093iA03p01871.

Rankin, R., J. C. Samson, and V. T. Tikhonchuk (1999), Parallel electric fields in dispersive Alfvén waves in the dipolar magnetosphere, Geophys. Res. Lett., 26, 3601-3604, doi:10.1029/1999GL010715.

Robinson, R., R. Vondrak, K. Miller, T. Dabbs, and D. Hardy (1987), On calculating ionospheric conductances from the flux and energy of precipitating electrons, J. Geophys. Res., 92, 2565-2569, doi:10.1029/ JA092iA03p02565.

Rothwell, P. L., M. B. Silevitch, and L. P. Block (1984), A model for the propagation of the westward traveling surge, J. Geophys. Res., 89, 8941-8948, doi:10.1029/JA089iA10p08941.

Russell, C. T., P. J. Chi, D. J. Dearborn, Y. S. Ge, B. Kuo-Tiong, J. D. Means, D. R. Pierce, K. M. Rowe, and R. C. Snare (2008), THEMIS ground-based magnetometers, Space Sci. Rev., 141, 389-412, doi:10.1007/s11214-008-9337-0.

Sakanoi, T., H. Fukunishi, and T. Mukai (1995), Relationship between field-aligned currents and inverted-V parallel potential drops observed at midlatitudes, J. Geophys. Res., 100, 19,343-19,360, doi:10.1029/ 95JA01285.

Sakanoi, T., S. Okano, Y. Obuchi, T. Kobayashi, M. Ejiri, K. Asamura, and M. Hirahara (2003), Development of the multi-spectral auroral camera onboard the index satellite, Adv. Space Res., 32, 379-384, doi:10.1016/ S0273-1177(03)90276-6.

Samson, J. C., J. A. Jacobs, and G. Rostoker (1971), Latitude-dependent characteristics of long-period geomagnetic micropulsations, J. Geophys. Res., 76, 3675-3683, doi:10.1029/JA076i016p03675.

Sato, T. (1978), A theory of quiet auroral arcs, J. Geophys. Res., 83, 1042-1048, doi:10.1029/JA083iA03p01042.

Sergeev, V. A., et al. (2000), Plasma sheet ion injections into the auroral bulge: Correlative study of spacecraft and ground observations, J. Geophys. Res., 105, 18,465-18,481, doi:10.1029/1999JA900435.

Seyler, C. H. (1990), A mathematical model of the structure and evolution of small-scale discrete auroral arcs, J. Geophys. Res., 95, 17,199-17,215, doi:10.1029/JA095iA10p17199.

Stasiewicz, K., et al. (2000), Small scale Alfvénic structure in the aurora, Space Sci. Rev., 92, 423-533, doi:10.1023/A:1005207202143.

Stenbaek-Nielsen, H., T. Hallinan, D. Osborne, J. Kimball, C. Chaston, J. McFadden, G. Delory, M. Temerin, and C. Carlson (1998), Aircraft observations conjugate to FAST, Geophys. Res. Lett., 25, 2073-2076, doi:10.1029/98GL01058.

Strangeway, R. J., et al. (1998), FAST observations of VLF waves in the auroral zone: Evidence for very low plasma densities, Geophys. Res. Lett., 25, 2065-2068, doi:10.1029/98GL00664.

Streltsov, A. V., and W. Lotko (1995), Dispersive field line resonances on auroral field lines, J. Geophys. Res., 100, 19,457-19,472, doi:10.1029/ 95JA01553.

Takahashi, K., R. E. Denton, and D. Gallegher (2002), Toroidal wave frequency at $\mathrm{L}=6-10$ : Active Magnetospheric Tracer Explorer/CCE observations and comparison with theoretical model, J. Geophys. Res., 107(A2), 1020, doi:10.1029/2001JA000197. 
Tsyganenko, N. (1989), A magnetospheric magnetic field model with a warped tail current sheet, Planet. Space Sci., 37, 5-20, doi:10.1016/ 0032-0633(89)90066-4

Wahlund, J.-E., et al. (1994), On ion acoustic turbulence and the nonlinear evolution of kinetic Alfvén waves in aurora, Geophys. Res. Lett., 21, 1831-1834, doi:10.1029/94GL01289.

Wahlund, J.-E., et al. (1998), Broadband ELF plasma emission during auroral emission: 1. Slow ion acoustic waves, J. Geophys. Res., 103, 4343-4375, doi:10.1029/97JA02008.

Weygand, J. M., O. Amm, V. Angelopoulos, S. E. Milan, A. Grocott, H. Gleisner, and C. Stolle (2012), Comparison between SuperDARN flow vectors and equivalent ionospheric currents from ground magnetometer arrays, J. Geophys. Res., 117, A05325, doi:10.1029/2011JA017407.

Williams, P. J. S., C. F. del Pozo, I. Hiscock, and R. Fallows (1998), Velocity of auroral arcs drifting equatorward from the polar cap, Ann. Geophys., 16, 1322, doi:10.1007/s00585-998-1322-0.

Wygant, J. R., et al. (2000), Polar spacecraft based comparisons of intense electric fields and Poynting flux near and within the plasma sheet-tail lobe boundary to UVI images: An energy source for the aurora, J. Geophys. Res., 105, 18,675-18,692, doi:10.1029/1999JA900500. 